VECC-NEX-98002

\title{
A Preshower Photon Multiplicity Detector for the WA98 Experiment
}

\author{
M.M. Aggarwal ${ }^{a}$, A. Agnihotri ${ }^{b}$, Z. Ahammed ${ }^{c}$, P.V.K.S. Baba ${ }^{d}$, S.K. Badyal ${ }^{d}$, K.B. \\ Bhalla $^{b}$, V.S. Bhatia ${ }^{a}$, S. Chattopadhyay ${ }^{c}$, A.C. Das ${ }^{c, 1}$, M.R. Dutta Majumdar ${ }^{c}$, M.S. \\ Ganti $^{c}$, T.K. Ghosh ${ }^{c, 2}$, S.K. Gupta ${ }^{b}$, H.H. Gutbrod ${ }^{g}$, S. Kachroo ${ }^{d}$, B.W. Kolb ${ }^{e}$, V. Kumar ${ }^{b}$, \\ I. Langbein ${ }^{e, 3}$, D.P. Mahapatra ${ }^{f}$, G.C. Mishra ${ }^{f}$, D.S. Mukhopadhyay ${ }^{c}$, B.K. Nandi ${ }^{f}$, S.K. \\ Nayak $^{f}$, T.K. Nayak ${ }^{c}$, M.L. Purschke ${ }^{e, 4}$, S. Raniwala ${ }^{b}$, V.S. Ramamurthy ${ }^{f, 5}$, N.K. Rao ${ }^{d}$, \\ S.S. Sambyal ${ }^{d}$, B.C. Sinha ${ }^{c}$, M.D. Trivedi ${ }^{c}$, J. Urbahn ${ }^{e}$, Y.P. Viyogi ${ }^{c, *}$ \\ ${ }^{a}$ University of Panjab, Chandigarh 160014, India \\ ${ }^{b}$ University of Rajasthan, Jaipur 302004, Rajasthan, India \\ ${ }^{c}$ Variable Energy Cyclotron Centre, Calcutta 700064, India \\ ${ }^{d}$ University of Jammu, Jammu 180001, India \\ ${ }^{e}$ Gesellschaft für Schwerionenforschung (GSI), D-64220 Darmstadt, Germany \\ ${ }^{f}$ Institute of Physics, Bhubaneswar 751005, India \\ ${ }^{g}$ SUBATECH, Ecole de Mines, F-44070 Nantes, France \\ * Corresponding author's address : \\ Variable Energy Cyclotron Centre \\ 1/AF, Bidhan Nagar, Calcutta 700064 (India) \\ tel : +91333371230, fax : +91333346871 \\ e-mail : viyogi@vecdec.veccal.ernet.in \\ ${ }^{1}$ Present address : Ohio State University, Columbus, Ohio, USA \\ ${ }^{2}$ Present address : KVI, Univ. of Groningen, Groningen, the Netherlands \\ ${ }^{3}$ Present address: IMK, FZK, D-76021 Karlsruhe, Germany \\ ${ }^{4}$ Present Address : BNL, Upton, New York, USA \\ ${ }^{5}$ Present Address : Department of Science and Technology, New Delhi 110016
}




\begin{abstract}
A high granularity preshower detector has been fabricated and installed in the WA98 Experiment at the CERN SPS for measuring the spatial distribution of photons produced in the forward region in lead ion induced interactions. Photons are counted by detecting the preshower signal in plastic scintillator pads placed behind a 3 radiation length thick lead converter and applying a threshold on the scintillator signal to reject the minimum ionizing particles. Techniques to improve the imaging of the fibre and performance of the detector in the high multiplicity environment of lead-lead collisions are described. Using Monte-Carlo simulation methods and test beam data of $\pi^{-}$and $e^{-}$at various energies the photon counting efficiency is estimated to be $68 \%$ for central and $73 \%$ for peripheral $\mathrm{Pb}+\mathrm{Pb}$ collisions.
\end{abstract}

PACS : 24.85.+p, 25.75.-q

Keywords: Photon Multiplicity Detector, Quark Gluon Plasma, WA98 Experiment, Preshower Detector

\title{
1 Introduction
}

The primary goal of ultra-relativistic heavy ion collision (URHIC) studies is to investigate the properties of matter at high energy density and high temperature. These studies provide information on the dynamics of multiparticle production mechanism and the phase transition from hadronic matter to Quark-Gluon Plasma (QGP). Several hadronic and leptonic signatures have been proposed to search for the QGP phase transition. Because of their weakly interacting nature, photons are supposed to be the carriers of information about the initial state of matter[1]. Recently, URHIC experiments have drawn wide attention for the study of QCD vacuum through the search of disoriented chiral condensates (DCC). Formation of DCC may lead to anomalous fluctuation of neutral pion fraction [2], leading to large discrepancies in the relative number of emitted charged particles and photons. This is analogous to the Centauro and the anti-Centauro types of events observed in cosmic ray experiments [3]. The study of isospin fluctuations entails the measurement of multiplicities of charged particles and photons. It is therefore important to study photon production in nuclear collisions over a wide range of the phase space.

A preferred technique for the detailed measurement of photons is to use electromagnetic calorimeters, which are usually 15-20 radiation lengths $\left(\mathrm{X}_{0}\right)$ deep [4]. Here one measures the energies and angles of emission of individual photons. However the large particle density in the forward hemisphere in URHIC experiments precludes the use of deep calorimeters because of enormous overlap of fully developed showers. The use of a preshower detector for measuring the multiplicity of photons in the forward hemisphere in $\mathrm{S}+\mathrm{Au}$ collisions at the SPS energy has already been demonstrated in the WA93 experiment [5]. It was shown that using lead converter of thickness $3 \mathrm{X}_{0}$, the preshower photon multiplicity detector (PMD) can be designed to handle the particle density at a moderate 
distance of 10 meters from the target, the resulting photon counting efficiency was $\sim 65 \%$ for central collisions.

In the present article we describe the implementation of a preshower PMD in the much higher particle density environment of $\mathrm{Pb}+\mathrm{Pb}$ collisions in the WA98 experiment [6] at the SPS energy. The PMD employed plastic scintillator pads to detect the preshower signal and wavelength shifting (WLS) plastic optical fibres to transport scintillation light to the image intensifier (II) and CCD readout cameras. It was fabricated and installed in the experiment in 1994. Simulation calculations for optimizing the phase space coverage and granularity of the detector are discussed in the next section. Results on R\&D efforts to improve the performance of the PMD are presented in section 3. The method of fabrication and quality control of the WA98 PMD is discussed in section 4. The performance of the detector in the test beam is described in section 5. Parametrization of the PMD necessary for simulating the effects of the entire detector are described in section 6 . The method used for photon counting is described in section 7. Results on the estimation of the efficiency of photon counting and the purity of the photon sample are also described here. A summary is given in the last section.

\section{Simulation Results and Detector Layout}

The main objective for the simulation studies in designing the preshower detector has been the optimization of converter thickness and granularity to handle large particle densities. A major constraint was the limited number of readout cameras (image intensifier and CCD camera systems) available from the UA2 experiment [7]. The role of converter thickness was extensively studied during the design of WA93 PMD and the optimized thickness was found to be $3 \mathrm{X}_{0}$. Compared to $\mathrm{S}+\mathrm{Au}$ collisions in the WA93 experiment, the particle density expected in $\mathrm{Pb}+\mathrm{Pb}$ collisions at the SPS energy was much higher. Hence a detailed simulation study was performed to optimize the pad sizes and pseudorapidity coverage of the detector using VENUS 4.12 event generator with default parameter settings [8] along with the GEANT 3.21 detector simulation package [9].

Fig. 1 1 shows the particle density $\left(\right.$ photons $/ \mathrm{cm}^{2}$ ) in the forward hemisphere in central $(b<2 \mathrm{fm}) \mathrm{Pb}+\mathrm{Pb}$ collisions at $158 \cdot A \mathrm{GeV}$ as a function of pseudorapidity $(\eta)$ at distances of $10 \mathrm{~m}$ and $20 \mathrm{~m}$ from the target as given by VENUS. The particle density in central $200 \cdot A \mathrm{GeV} \mathrm{S}+\mathrm{Au}$ collisions at $10 \mathrm{~m}$ from the target (corresponding to the location of the PMD in the WA93 experiment) is also superimposed for comparison. It is observed that (a) even at the lowest $\eta$ - range under consideration, particle density in $\mathrm{Pb}+\mathrm{Pb}$ collisions is comparable to that in the WA93 case only at a much larger distance from the target, and (b) even at such a large distance the density is $2-3$ times higher at larger pseudorapidities, necessitating the use of smaller pads compared to those of the WA93 PMD in order to minimize the shower overlap.

In addition to the above requirements for handling large particle densities, the detector layout and design was influenced by the following considerations : (a) it would be useful to extend the detector coverage a little into the backward hemisphere $(\eta<2.9)$ 
to determine the peak of the pseudorapidity distribution properly, (b) the PMD with its associated lead converter plates and support structure should not spray appreciable secondary particles onto the lead glass calorimeter sitting just behind it; (c) the PMD should have full azimuthal coverage for the largest angular region possible; and (d) the PMD should not receive appreciable spray of secondaries from the upstream detectors and their support structures.

Studies of occupancy (ratio of the number of hit pads to the total number of pads) and multiple-hit probability (as defined in [5]) were carried out using VENUS and GEANT for square pads of different sizes, considering only the PMD placed in air. The results are shown in Fig. 2 for possible configurations using $15 \mathrm{~mm}$ and $25 \mathrm{~mm}$ pads over the entire detector placed at $20 \mathrm{~m}$ from the target. The results suggest that it was not possible to use the large pad sizes throughout the detector because of very high occupancy and correspondingly large shower overlap. Using smaller pad sizes throughout was also ruled out because of the requirement of very large number of readout cameras. As the particle density varied by a factor of more than 2 over the region of interest, it was considered appropriate to use different pad sizes over different regions. Because of the large size of the detector and the associated difficulties in handling the detector during assembly, transport and installation, it was not convenient to have a single light-tight enclosure like that of the WA93 PMD. Therefore a modular approach was adopted, entire detector being subdivided into box modules. Each box module was a light-tight enclosure, housing pads of a given size to be readout using one II + CCD camera system. It contained pads in a matrix of 38 rows and 50 columns. Thus the size of a box module was determined by the size of the pads within it, varying from about $75 \mathrm{~cm}$ to $125 \mathrm{~cm}$ in length and proportionately in width. This could be easily handled during assembly and installation.

Several combinations of pad sizes and number of box modules of each kind were tried keeping the phase space coverage and the total number of readout units within the design goals. The final optimized layout of the box modules is shown in Fig. 3. This contains square pads of sizes $15 \mathrm{~mm}, 20 \mathrm{~mm}$ and $25 \mathrm{~mm}$, smaller pads being placed in the innermost regions. The box modules 21 and 22 as shown in Fig. 3 were made with $23 \mathrm{~mm}$ pads to cover the gaps and achieve full azimuthal coverage without much loss of granularity. This resulted in a geometrically compact arrangement. The PMD was finally located at $21.5 \mathrm{~m}$ from the target. The detector extends to approx. $21 \mathrm{~m}^{2}$ in area and covers the pseudorapidity region $2.5 \leq \eta \leq 4.2$, of which the region $3.2 \leq \eta \leq 4.0$ has full azimuthal coverage. With this arrangement for the entire PMD, consisting of 28 box modules, the occupancy was well within $10 \%$ and multiple hit probability within $5 \%$, being almost uniform over the entire surface of the detector (see Fig. 2).

\section{$3 \quad$ R\&D on Technology Improvement}

The basic technique of fabrication and assembly of the PMD in various steps, viz., cutting and drilling of scintillator pads, coupling the WLS fibre, assembly of pads in light-tight enclosures and the use of perforated fibre end coupler (FEC) plate for arranging the 
matrix of fibre bundles was similar to that used in the case of WA93 PMD [5]. Several improvements were incorporated at various steps during the fabrication and assembly of pads so that attenuation of scintillation light passing through the fibre was minimized and the image of the fibre on the CCD surface was focussed onto fewer number of pixels. R\&D results on these technological improvements are described below. Preliminary results have been reported earlier [10].

\subsection{Techniques for minimizing light attenuation}

\section{(i) Use of short length WLS fibre.}

The emission peak of the WLS (BCF91A) fibre used in the present detector lies at $480 \mathrm{~nm}$. A substantial fraction of light around this wavelength is lost by self-absorption in the dye molecules of the WLS fibre after traversing a length of about $20 \mathrm{~cm}$. Self absorption also results in shift of the mean wavelength to a larger value where the quantum efficiency of the S20 photocathode of the image intensifier is lower. Thus there is a substantial decrease in the detector signal at the end of the readout chain if long lengths of the WLS fibre are used, as in the case of WA93 PMD.

The length of the fibre required to transport light from the scintillator pads to the readout device in the modular design discussed earlier varies from $1.5 \mathrm{~m}$ for box modules containing $15 \mathrm{~mm}$ pads to $2.5 \mathrm{~m}$ for those containing $25 \mathrm{~mm}$ pads. Such large lengths are required to prevent the formation of loops of smaller radii in the fibre, which might lead to loss of light in the long run.

While WLS fibre must be used inside the scintillator pad, it is not necessary to continue with the same fibre outside for long lengths. In order to improve light transmission, only $8 \mathrm{~cm}$ long WLS fibres were used. These were joined with clear plastic fibres. For mechanical protection and also to reduce the numerical aperture at the exit end, clear fibres with extra-mural absorber (EMA) coating were used. As compared to the use of PVC sleeves to protect the WLS fibres, as in the WA93 case, the EMA coating was preferred as the overall thickness of the fibre remained small and managing the bundle of 1900 fibres became relatively easy.

(ii) Splicing of clear fibre to the WLS fibre.

The WLS fibre and the clear fibre were joined using a specially designed splicing jig shown in Fig. 4. It consists of two $1 \mathrm{~mm}$ thick mating jaws made of copper, one movable and the other fixed, attached with good thermal contact to a copper block. The copper block is fitted with a 50 watt heater cartridge and a temperature sensor. The jaws have small semi-circular cuts on the mating edges and in closed position form a circular opening of $1.5 \mathrm{~mm}$ diameter. The movable jaw is spring loaded and remains in normally open position. It can be closed by a foot operated pedal arrangement. 
The procedure for splicing is as follows :

The ends of the clear and the WLS fibres to be joined are carefully cut normal to the axis. EMA coating is removed from about $2 \mathrm{~cm}$ length of the clear fibre end. The prepared ends of WLS and clear fibres are inserted into a $1 \mathrm{~cm}$ long thin walled glass capillary tube of $1.02 \mathrm{~mm}$ inner diameter and $1.5 \mathrm{~mm}$ outer diameter. The capillary tube is introduced into the cutout of the heated $\left(150{ }^{\circ} \mathrm{C}\right)$ copper jaws (guided by a groove on the teflon support platform of the jig). The jaws are closed and the fibre junction is heated. After a pre-calibrated time (usually about a few seconds) the jaws are released. The resulting spliced joint is tested for mechanical strength by hanging a load of $500 \mathrm{~g}$. The joints are also subjected to light leakage test by physically viewing the joint through a 20X microviewer. Bad joints which do not have sufficient strength or which show rings of light around the splicing area are rejected. The short capillary tube is retained over the splice and fixed with a drop of glue to improve the strength of the spliced joint.

The fraction of light transmitted through the spliced joint (called splicing efficiency in the present article) was measured using an LED/PIN diode setup similar to that used to characterize the fibres in the case of WA93 PMD. For this measurement a short $(8$ $\mathrm{cm})$ piece of clear fibre was spliced to another long $(192 \mathrm{~cm})$ piece of the same fibre. In this system light was launched longitudinally into the short section and the light output measured at the other end. The result is shown in Fig. 0 for a sample of spliced pieces. Mean splicing efficiency of more than $85 \%$ was achieved even with such an inexpensive jig and a simple splicing procedure. The width of the distribution, which is a measure of the piece-to-piece fluctuation in light transmission through the spliced joint, is found to be less than $5 \%$.

For a quantitative estimate of the improvement of the detector signal after the use of splicing technique, the scintillator pad-fibre combination was irradiated with ${ }^{106} \mathrm{Ru} \beta$ source and the detector signal measured using a photomultiplier tube (PMT) setup. The mean signal for different sets of samples are shown in Fig. 6 for the case of full length WLS fibre (open circles) and the short WLS fibre spliced with long clear fibre (open squares). Systematic errors, resulting from coupling of the fibre to the PMT and the end finishing, are of the order of $10 \%$. It is observed that the signal decreases rapidly with increasing length for the full length WLS fibre case. In contrast, the drop in signal with increasing fibre length for the case of short length WLS fibre coupled with long length clear fibre is rather slow. In comparison to the full length WLS case, the signal for the spliced case increases marginally for small total length and by more than $50 \%$ for larger total length of the fibre, compensating for the sharp drop in the earlier case. Thus the use of clear fibre makes the pad output almost uniform for different fibre sizes.

(iii) Painting the tip of the fibre.

Light traversing within the WLS fibre towards the fibre end, which is inside the scintillator pad, emerges within the pad and is lost because of multiple reflection. A part of this light can be sent back into the fibre if the tip is shining and painted with scintillator grade reflector paint. 
The results on improvement in light collection arising from the use of above procedures are also presented in Fig. 6. Improvement due to tip painting is found to be significant only when the total fibre length is small. For larger lengths of the fibre, the improvement is only marginal. However for maintaining the uniformity of pad response for various fibre lengths, this procedure was introduced during the fabrication of the pads.

\subsection{Improvement of fibre imaging in readout}

The image of the fibre on the CCD surface spreads over a set of pixels. The number of pixels per fibre is not uniform, being smaller near the boundaries than in the middle. Because of imperfections in opto-electronic readout and approximations used in the schemes for generating pixel-to-fibre maps [5], some of the pixels belonging to a particular fibre may be assigned to neighbouring fibres, giving signal in several fibres even though light originated in only one pad. Thus light "leaks out" to neighbouring fibres. This effect has been discussed in detail in the case of WA93 PMD [5]. Another source of this light leakage is the stray off-axis light in the fibre incident at the interface of the entrance window of the II chain. This off-axis light tends to broaden the image of the fibre at the CCD surface. To use the II + CCD device in the high multiplicity environment of $\mathrm{Pb}+\mathrm{Pb}$ collisions, the quality of the fibre image on the CCD must be improved and the light leakage outside the assigned pixels minimized. Several techniques were investigated to reduce the effect of off-axis light in the fibre up to the surface of contact with the readout system.

Off-axis light traveling in the fibre is found to be substantially reduced by coating the cladding surface of the fibre. This results in reduced numerical aperture. Light from the fibre enters the II system through a glass window at the surface of contact of the FEC plate and the II system. If the fibre bundle is embedded in a transparent medium (as it was in the case of WA93 PMD), light reflected from the glass surface may undergo multiple reflection and re-enter the II system, thus increasing cross-talk and broadening the image of the fibre. This will lead to further increase in shower overlap in a high multiplicity environment. This effect can be reduced by applying black paint over the cladding surfaces near the exit end of the fibre bundle so that the entire bundle becomes an opaque mass. The results of the effects of applying various paints to the fibres are presented in Table I in terms of the effect on the measured numerical aperture.

The numerical aperture reduces slightly for larger length of the fibre because of loss of some off-axis light at the cladding-air interface over the length of the fibre. This is evident by comparing the values of numerical aperture for $8 \mathrm{~cm}$ and $200 \mathrm{~cm}$ long WLS fibres. Application of paint to the fibre (on the cladding surface) also reduces the numerical aperture by cutting down off-axis light, black paint is more effective in this regard. Splicing with clear coated fibre is much more effective in reducing the numerical aperture at the exit end. With the removal of off-axis light and consequent reduction in numerical aperture the signal of the pad may also decrease, but the image of the fibre on the CCD surface should be sharper. Final results on improvement in imaging of the fibre on the CCD surface are discussed in sections 5.1.2 and 5.3.2. 
TABLE I

Numerical aperture of plastic optical fibres in various conditions.

\begin{tabular}{||c|c|c|c||}
\hline \hline $\begin{array}{c}\text { WLS fibre } \\
\text { length }(\mathrm{cm})\end{array}$ & $\begin{array}{c}\text { Clear fibre } \\
\text { length }(\mathrm{cm})\end{array}$ & $\begin{array}{c}\text { Fibre surface at } \\
\text { the exit end }\end{array}$ & $\begin{array}{c}\text { Numerical } \\
\text { Aperture }\end{array}$ \\
\hline \hline 8.0 & - & - & 0.67 \\
200.0 & - & - & 0.60 \\
\hline 8.0 & - & White paint & 0.64 \\
8.0 & - & Black paint & 0.62 \\
\hline 8.0 & 18.0 & Bare* & 0.61 \\
8.0 & 36.0 & Bare* $^{*}$ & 0.52 \\
8.0 & 200.0 & Bare* $^{*}$ & 0.50 \\
\hline \hline
\end{tabular}

* For these measurements, "bare" denotes the condition that EMA coating was removed from about $5 \mathrm{~mm}$ length of the clear fibre near the end.

\section{WA98 Photon Multiplicity Detector}

\subsection{Mechanical description}

\subsubsection{Fabrication of pads}

The plastic scintillator sheets (3 mm thick BC-400) were cut into square pads of sizes 15 $\mathrm{mm}, 20 \mathrm{~mm}, 23 \mathrm{~mm}$ and $25 \mathrm{~mm}$ by using proper tools and cooling procedures developed earlier during the fabrication of WA93 PMD. Blind diagonal holes of $1.1 \mathrm{~mm}$ diameter were drilled into the pads. After proper cleaning and drying of pads, a calibrated amount of optical glue (BC600) was injected into the hole. Short $(8 \mathrm{~cm})$ pieces of WLS fibre, with reflector paint applied to one tip, were inserted into the holes and lowered slowly till the end without trapping air bubbles. After allowing for a curing time of about 24 hours, the pads were painted with a thick coat of scintillator grade reflector paint. Splicing of the clear coated fibres of desired length were then carried out according to the procedure described in the previous section. Small $(10 \mathrm{~cm})$ pieces of black PVC sleeves were then fastened over the fibre near the pad end to protect the WLS fibre section and the spliced joint from mechanical damage and to prevent from picking up stray light. All these operations were carried out in a humidity and dust controlled environment.

Control on the splicing operation and check on reproducibility was carried out by splicing samples of two clear fibre pieces and measuring the splicing efficiency at regular intervals. For proper quality control and to maintain close uniformity among the pads, sample detector pads were tested in well calibrated $\beta$-ray test setup using ${ }^{106} \mathrm{Ru}$ source. Suitable sampling was done in batches for ensuring proper quality of pads of different

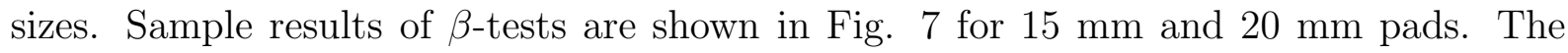


$\mathrm{x}$-axis denotes the peak channel number of the digitized PMT signal. The uniformity of the pads is better than $10 \%$. The response of pads of different sizes is also very similar. This makes the entire PMD uniform in response to minimum ionizing particles.

\subsubsection{Assembly of detector box modules}

A typical detector box module, shown in Fig. 8, consists of two parts : (1) a light tight pad enclosure of height $125 \mathrm{~mm}$, made of $1.2 \mathrm{~mm}$ thick steel sheet, to house the scintillator pads and (2) a camera enclosure (700 mm length, $300 \mathrm{~mm}$ width, $300 \mathrm{~mm}$ height) to house the II and CCD system. The dimensions of the pad enclosures differ according to the size of the pads. The camera enclosure was identical for all the modules. This was fixed on top of the pad enclosure with insulating studs for electrical isolation. The top lid of the pad enclosure had a cutout for the fibres to pass through. A light-tight rubber bellow, with an aluminum collar and a fibre-end-coupler (FEC) plate, joined the pad enclosure to the camera enclosure. The bellow assembly provided a flexible neck for the bundle of 1900 fibres and formed an extension of the light tight enclosure up to the interface of the II system.

A brass sheet of thickness $0.1 \mathrm{~mm}$, die pressed in the form of staircase steps, was attached to the bottom face of the detector encloser to fix pads in rows and columns leaving room to take out the fibre bundle above the pad planes. Pads were fixed to the brass steps using colourless Silastic (RTV732) sealant. Free ends of the fibres were taken through the bellows to the FEC plate. The FEC plates having $1.1 \mathrm{~mm}$ diameter holes in a matrix of 38 rows and 50 columns were identical to those used in the WA93 PMD. Fibres were inserted into these holes maintaining strict one-to-one correspondence between the pad coordinates in the box and the coordinates of the corresponding fibre on the FEC plate.

The fibre coordinate at the top right corner of the FEC plate, as viewed from the polished surface, is denoted $(01,01)$. The corresponding position on the pad enclosure was marked for proper installation and identification with pixel coordinates on the CCD during generation of pixel-to-fibre maps.

After proper insertion of the fibres in the FEC plate, the bundle was trimmed, potted, machined and then hand polished to a mirror finish to achieve good light coupling with the glass window of the readout device. For potting the fibre bundle a 3-component resin consisting of CIBA-GEIGY AY103 araldite, HY956 hardener and DW0137 black additive was used. The black component made the FEC plate opaque to light reflected from the glass window of the readout device. This prevented multiple reflection of light and re-entry into the II system, thus minimizing cross-talk and broadening of the image of the fibre on the CCD surface. Finally the top lid of the pad enclosure was fixed in place with a black silicon sealant. This made the entire enclosure light-tight.

The front window of the II + CCD camera was coupled to the polished end of the fibre bundle on the FEC plate and held in position with long screws, as shown in Fig.

9(a). A connector panel at the side of the camera enclosure, shown in Fig. 9(b), was used 
to supply voltages to the II chain and the CCD electronics and to carry signals from the CCD. This also provided feedthrus for the optical fibre link from the xenon flasher to the local homogenizer described below.

\subsubsection{Fiducial fibres}

In each box module there were 101 pads which had one additional clear fibre inserted for selective illumination. These pads, called fiducial pads, were randomly but uniformly distributed over the matrix. The other ends of the clear fibres of these fiducial pads (referred to as fiducial fibres) were bundled and potted with an optical glue into a small perspex disk of $20 \mathrm{~mm}$ diameter fitted with a brass collar and brought out through a hole on the side of the rubber bellow. A $250 \mathrm{~mm}$ long and $20 \mathrm{~mm}$ diameter perspex homogenizer rod was coupled to the fiducial fibre bundle. This system was enclosed in a black plastic tubular housing to make it light tight. It was situated within the camera enclosure of each box module. The other end of the homogenizer was connected to an optical fibre through the connector panel in the camera enclosure. This fibre formed one of the branches of a light distribution system and was optically connected to the xenon flasher device.

Light flashes for the fiducial fibres in all the box modules of the PMD were generated by an externally triggered flasher system as shown in Fig. 10. This consisted of a high efficiency xenon lamp (Hamamatsu L2445) having a stable arc size of $8.0 \mathrm{~mm} \times 1.5 \mathrm{~mm}$, a perspex homogenizer rod of $20 \mathrm{~mm}$ diameter and $300 \mathrm{~mm}$ length and a set of plastic fibres (1 mm dia) to transmit light to various detector box modules.

Measurement of the coordinates of the fiducial fibres in terms of pixel coordinates on the CCD surface was necessary to generate the pixel-to-fibre map by the procedure described in Ref. [0].

\subsubsection{Support structure and detector installation}

The support structure for the PMD was made up of five sections of $6 \mathrm{~mm}$ thick steel plate welded together and reinforced at the boundary by rigid channels as shown in Fig. 11(a). The central section was perpendicular to the beam axis. The sections on the two sides and on the top and bottom were tilted inward by about $8^{\circ}$ in order to maintain a near-normal incidence for all regions of the detector. The entire support structure was fixed to a rigid stand fitted with rollers and could be moved longitudinally on rails to facilitate service access.

Lead converter plates were mounted on the steel plate of the support structure facing the target. A set of fiducial markers were also fixed on the support structure above the lead plates for proper geometrical alignment of the detector with respect to the beam axis.

Detector box modules were mounted on the back side of the support structure in accordance with the layout scheme shown in Fig. 3. Twelve box modules containing 15 
mm pads, four box modules containing $20 \mathrm{~mm}$ pads and two box modules containing 23 $\mathrm{mm}$ pads were mounted on the central section of the support structure. The side sections supported modules containing $25 \mathrm{~mm}$ pads and the top and bottom sections supported modules numbering 14, 15, 18 and 19. Fig. 11(b) shows the detector box modules and the camera enclosures. In the box modules mounted on the top and bottom sections, corners were cut and sets of $12 \times 12$ pads were removed so that the PMD could be moved very close to the lead glass calorimeter in the final position without mechanical interference from its massive support structure.

One set of cables carried high voltage from the counting area to a location near the detector. High voltage was then supplied to the individual cameras through a set of distribution boxes. All the low-voltage supplies for the CCD electronics were kept near the detector and their status was monitored remotely. The length of the signal cables from the detector to the digitizer was about $50 \mathrm{~m}$.

The CCD electronics board of the II system was cooled by a built-in fan. In case of malfunction of this fan, camera noise would increase due to rise in temperature. In order to monitor temperature variations of the CCD housing, temperature sensors (Analog Devices AD590) were attached to the enclosure close to the CCD chip and the temperatures of all the camera enclosures were monitored via a remote digital readout.

\subsection{Readout}

The II + CCD system of the UA2 experiment [7] has been used for the readout of the scintillator light signal as in the case of WA93 PMD. The 3-stage II chain provides light amplification of the order of 40000. The CCD has a total of 31600 pixels arranged in rows, each row having 218 pixels. A map assigning each pixel to a fibre, called the pixel-to-fibre map, is generated to transform the CCD pixel coordinates to the fibre coordinates of the PMD for clustering of preshower hits.

The CCD pixel charge is digitized using a custom-built FASTBUS module using 8-bit $20 \mathrm{MHz}$ flash ADCs. The reading of the FASTBUS module by the data acquisition system and the corresponding timing diagram have been discussed in detail in Ref. [5]. A free running clock of $1 \mu \mathrm{s}$ width was used to generate the clear signal in every $10 \mu$ s. This eliminated the CCD noise stored during the intermediate time. The time difference between the arrival of the clear clock and the event trigger was measured using a TDC having a range of $10 \mu \mathrm{s}$. This helped in rejecting events which were partially or fully cleared due to the arrival of the clear clock. Details of the event clean-up procedure will be described in subsequent publications.

The digitizer takes data in three different modes [5, 7] :

Mode 1: measures charges in all pixels without pedestal subtraction. This mode is used to generate data on the pedestal charge of the pixels and stored in the digitizer memory for use in Mode 2 and Mode 3.

Mode 2: this gives digitized pixel charge after pedestal subtraction. Only pixel charges above a preset hardware threshold, accompanied by the address, are transmitted during 
reading of the digitizer.

Mode 3: This gives fibre signal, i.e., sum of Mode 2 data for the set of pixels assigned to a fibre as given by the pixel-to-fibre map. Pixel-to-fibre map is loaded into the digitizer memory. Fibre number and number of fired pixels are transmitted together with the fibre sum. This produces very compact data format.

In addition to the readout of the CCDs with the FASTBUS digitizers it was possible to view the CCD images triggered by a timing generator in television mode (TV-mode). This mode was used to inspect visually the camera gain and noise and the alignment of pads to the test beam.

Small volumes of data, as generated during test beam and fiducial runs, were collected in Mode 2. In the main experiment the data volume of the PMD was large and hence it was necessary to use Mode 3 option only. The pixel-to-fibre maps were generated using fiducial data taken at regular intervals and loaded into the digitizer memory.

\section{Detector Characteristics and Test Beam Results}

The characteristics of the PMD, e.g., response to varying input light levels, minimum ionizing signal and preshower behaviour, were studied using test beams and Monte-Carlo simulations of known particle types $\left(\pi^{-}\right.$and $\left.e^{-}\right)$with different incident energies and also using fiducial lighting at various light intensities. The results will be described by using the terms "ADC", "pad ADC" and "cluster ADC" [5]. ADC is used here to denote the ADC channel number of the digitized data. Pad ADC denotes the sum of the ADC channel numbers of the pixels (digitized charge after pedestal subtraction) assigned to a particular pad (fibre), the term "cluster ADC" denotes the sum of the ADC channel numbers of all the pixels in a cluster in mode 2 or the sum of pad ADCs of all the pads in the cluster in mode 3 . The cluster may be that due to a hadron (and its secondaries) or a photon preshower or a lighted fiducial fibre.

\subsection{Results from fiducial lighting}

Studies with fiducial lighting were carried out for all the box modules fitted with readout cameras. The results are described below.

\subsubsection{Study of camera noise}

Using data on fiducial lighting the noise behaviour of the II + CCD readout cameras has been studied. In the fiducial pattern a control zone is selected in which a fibre is isolated from the neighbouring fiducial fibres at least by \pm 2 pads. In principle no signal is expected in this zone. These zones were studied very carefully for all the cameras. It was found that these zones contain mostly single pixel clusters with $\leq 4$ ADC channels, as was also found in the case of WA93 PMD. Mean ADC of the clusters in different cameras 
varied from 3.5 to 4.1. This noise is rejected in the offline analysis before processing the data for physics studies.

\subsubsection{Light leakage}

The finite resolution of the opto-electronic readout causes a distortion in the image of the rectangular pattern such that the assignment of pixels to a fibre is non-uniform, the number of pixels per fibre being lesser at the boundaries than in the central region of the CCD surface. In addition off-axis light in the fibre causes spreading of the image. This results in a fraction of the light from a particular fibre to be detected outside the set of pixels assigned to that fibre. The neighbouring fibres thus appear to give signal even though the corresponding pads have no light. This leads to artificial spreading of the image of a fibre due to the readout system. The fraction of light "leaking out" also depends on the intensity of input light [5]. It is therefore necessary to study the behaviour of light leakage to the surrounding fibres.

The leakage was studied by considering a set of doubly isolated fiducial fibres in fiducial data, there being only one fiducial fibre in a $5 \times 5$ matrix. Thus the effect of light leaking from the central fibre to the surroundings in a $3 \times 3$ matrix can be studied, as these fibres are not expected to be affected by light from other fiducial fibres. The input light level was varied by adjusting the intensity of the flasher system. The light leakage as a function of the intensity (represented by total ADC of the cluster) was found to be more in the readout direction at all light levels, as reported earlier [0]. The percentage of leakage (total ADC channel numbers in the surrounding fibres with respect to the cluster ADC), shown in Fig. 12, slowly increases and then saturates with increase in input light intensity. Minimum leakage at low intensity is $\sim 25 \%$ and the saturation value is $\sim 40 \%$.

It is also observed that the light leakage for the WA98 PMD is less than that of the WA93 PMD at all light levels. The saturation value is about $30 \%$ less compared to the WA93 case and occurs at much higher light levels (beyond 500 ADC channels). This demonstrates the improvements in the detector as described in section 3 .

\subsubsection{Spatial spreading of fibre images}

Light leakage discussed above causes the image of a fibre to spread to several neighbouring fibres. The number of extra fibres giving signal depends on the intensity of incident light. This has been studied in detail for several doubly isolated fiducial fibres. The mean probability of a number of extra fibres receiving leaked light is plotted in Fig. 13 for a set of light intensities, denoted by the cluster ADC values.

At lower light levels an average of 4 to 5 extra fibres are affected by the leaked light. The distribution is almost symmetric. As the light intensity increases, the maximum of the probability distribution shifts towards larger number of surrounding fibres and the distribution becomes asymmetric. Beyond a certain light intensity all the eight surrounding fibres receive leaked light. 
Because of unequal distance of perpendicular and diagonal fibres in a rectangular matrix and also because of the effect of higher charge collection along the readout direction, fractional distribution of leaked light into the surrounding fibres is not uniform. The distribution of leaked light into various neighbouring fibres has been studied using fiducial data at several light intensities.

TABLE II

Percentage distribution of leaked light to various fibres.

\begin{tabular}{|c|r|c|r|r|r|r|r|}
\hline Fibre No. $\begin{array}{r}\text { Fibres } \\
\text { Fibra }\end{array}$ & 2 & 3 & 4 & 5 & 6 & 7 & 8 \\
\hline 1 & 0 & 0 & 0 & 10 & 7 & 5 & 4 \\
2 & 0 & 0 & 15 & 17 & 14 & 10 & 11 \\
3 & 0 & 0 & 0 & 0 & 0 & 0 & 4 \\
4 & 40 & 35 & 32 & 28 & 31 & 30 & 27 \\
5 & 0 & 0 & 0 & 0 & 0 & 4 & 3 \\
6 & 0 & 20 & 16 & 13 & 8 & 10 & 13 \\
7 & 0 & 0 & 0 & 0 & 5 & 3 & 3 \\
8 & 60 & 45 & 37 & 32 & 35 & 38 & 35 \\
\hline
\end{tabular}

Fig. 14 displays the nomenclature for addressing the fibres around a fiducial one in a $3 \times 3$ matrix. Table II summarizes the results on the percentage of leaked light going into a given fibre for various cases of the number of extra fibres affected. It is found from Table II that fibres situated at the corners of the rectangular matrix receive least light. Among the other four fibres those in the horizontal row receive more light than the fibres vertically opposite the fiducial one. The fibre situated to the right of the fiducial (\# 8) receives maximum light in all the cases.

\section{$5.2 \quad$ Test beam setup}

The tests of the PMD were carried out in the X1 beam line at the CERN SPS using secondary beams of $e^{-}$and $\pi^{-}$of varying energies in the range $3-50 \mathrm{GeV}$. Due to the problem of enormous time required in exposing all the pads individually to the beams, only one box module of $15 \mathrm{~mm}$ pads was tested in the beam. Information on the behaviour of all the other box modules was obtained by comparing the results of fiducial lighting and Monte-Carlo simulations with those for the tested box module.

The box module was positioned vertically on a remote controlled movable stand in the X1 beam line. The initial position of the beam was fixed on a particular fiducial pad by viewing the image of the fibre in TV mode. The stand was then moved in suitable directions to bring other pads into the beam for tests. 
Triggering arrangement consisted of a set of three scintillator paddles. One scintillator paddle was placed in front of the beam exit and the other one at the detector box. The third one with a hole was placed in between these paddles. For a valid trigger, the two outer scintillators were required to be in coincidence between them and in anti-coincidence with the paddle having a hole.

Signals from Nitrogen and Helium filled Cerenkov counters placed upstream and a delay-line wire chamber placed in front of the detector were recorded for offline use. Cerenkov signals were used for the separation of electron and pion beams. The triggering allowed a small $(<1 \%)$ admixture of electrons in the pion sample. The beam profile was obtained from the wire chamber. The offline data analysis selected only those hits of the beam particles which were located in the central position of the wire chamber profile.

\subsection{Test results}

\subsubsection{Minimum Ionizing Particle (MIP) signal}

Most of the charged hadrons impinging on the PMD deposit energy equivalent to a minimum ionizing particle (MIP). A detailed knowledge of the MIP response on the PMD is necessary for parametrization of the detector simulation, for rejection of hadrons by applying a threshold on the cluster signal and for the estimation of photon counting efficiency [5.

The response of the pads to minimum ionizing particles was studied using $50 \mathrm{GeV}$ $\pi^{-}$beam without lead converter. All the MIP properties like the number of fibres affected and the percentage of light leaking to the surrounding fibres were studied using the $\pi^{-}$test data. About $30 \%$ of the MIP clusters are confined to one fibre (pad) only. A typical MIP spectrum is shown in Fig. 15. Describing the MIP spectrum by a Landau distribution, the mean is found to be $39 \pm 1$ ADC channels.

The single photoelectron response of the II + CCD system is studied by fitting multiple Poisson distributions to the MIP spectrum. The mean value of the first Poisson component (with $\mathrm{n}=1$ ) is found to be $12.2 \pm 1$ ADC channels, which represents the signal due to a single photoelectron produced at the first photocathode of II chain. This value is very close to that observed earlier in the case of the WA93 PMD, suggesting that the camera gains were very similar in the two cases. Considering the above value of the single photoelectron response, MIP signal is equivalent to $\sim 3.2$ photoelectrons. The number of photoelectrons in the $\beta$-spectrum of scintillator pads, measured during fabrication, was found to be $\sim 4[10]$. The above two results are consistent with each other considering the fact that (a) some reduction in light output would have resulted in the assembled version of the detector because of the black paint, and (b) the energy deposition in $\beta$-source tests is not truly MIP-like, but slightly higher, because of contributions from very low energy $\gamma$-rays in the radioactive source.

The MIP response suggests that the light output of the WA98 PMD is higher than that of the WA93 PMD [5]. However, the effect is not as large as would be suggested from 
Fig. 6. The reason can again be traced to the reduction in off-axis light by the paints on the fibre surface and at the ends. Considering the requirement of dynamic range, as discussed in [5], the present value of the MIP signal is quite satisfactory, a large increase would have resulted in saturation of the pad signal in many cases.

\subsubsection{Preshower characteristics}

Preshower characteristics of the PMD, like the energy deposition and transverse spread (number of pads fired), have been studied using a $3 \mathrm{X}_{0}$ thick lead converter placed in front of the detector box module bombarded with electrons of varying energies in the range 3 $\mathrm{GeV}$ to $50 \mathrm{GeV}$.

Data analysis was carried out after noise filtration in mode 2 data and transformation to mode 3 (fibre or pad coordinates) by using the appropriate pixel-to-fibre map generated from the fiducial data taken during that period. A zone of $10 \times 10$ pads around the centroid of the beam was chosen in the pad space. The pad hits were analyzed in two ways to study the systematic uncertainty in the mean number of pads fired and the mean energy deposition for different electron energies. In the first method total cluster signal was obtained simply by adding the signals in hit pads in the selected zone. In the second method the cluster signal was obtained by using proper clustering algorithm developed earlier [5]. The cluster signal obtained from the two methods differ by less than $5 \%$, the clustering method yielding a slightly lower value. The average values of the two methods have been taken for the analysis.

The detector simulation has been carried out using GEANT for single particle $\pi^{-}$ and $e^{-}$at specified energies. The systematic uncertainty in the mean energy deposition due to the variation in cut-off energies of the secondaries and $\delta$-rays produced in the lead converter has been studied in detail. The cut-off parameters have been optimized to values which do not affect the results on energy deposition and also minimize computation time. Discussions on this are presented below.

\section{(a) Transverse shower spread}

The number of pads hit is a measure of the transverse shower spread. Fig. 16(a) shows the variation of mean number of pads (of size $15 \mathrm{~mm}$ ) for different electron energies. The GEANT simulation results are also superimposed for comparison. It is found that the total number of pads fired in the test beam is more than that fired in the simulation. This is mainly because of light leakage arising from imperfections in pixel-to-fibre maps and the distortion of the image on the CCD surface as discussed earlier.

It is observed that the mean number of pads fired is much less compared to those in the WA93 PMD for all electron energies, even though the pad area in the present case is almost half of that in the WA93 case. A comparison of the GEANT simulation and test beam results of WA93 and WA98 PMDs is presented in Table III. It is observed that while the number of pads fired in test beam data at any particular electron energy was more than four times that of GEANT simulation in the WA93 case, in the present case 
TABLE III

Comparison of average number of pads fired in GEANT simulation and test data for various electron energies.

\begin{tabular}{|c|c|c|c|c|}
\hline \multirow{2}{*}{$\begin{array}{c}\text { Energy } \\
(\mathrm{GeV})\end{array}$} & \multicolumn{2}{|c|}{$\begin{array}{c}\text { WA93 PMD } \\
(20 \mathrm{~mm} \text { pads })\end{array}$} & \multicolumn{2}{c|}{$\begin{array}{c}\text { WA98 PMD } \\
(15 \mathrm{~mm} \text { pads })\end{array}$} \\
\cline { 2 - 5 } & Simulation & Test beam & Simulation & Test beam \\
\hline 3 & 3 & 16 & 4 & 7 \\
5 & 4 & 18 & 5 & 9 \\
10 & 4.5 & 19 & 6 & 13 \\
20 & 5 & 21 & 7 & 15 \\
\hline
\end{tabular}

the number of pads increases only by a factor of about two in going from GEANT to test beam results. The transverse spread of the preshower is greatly reduced because of the technological improvements in fibre imaging. It is to be noted that in the case of the WA93 PMD the potting of the FEC plate having the fibre bundle was done using a transparent resin, while in present case it was black resin.

(b) Energy deposition

The mean cluster ADC values are plotted as a function of incident electron energy in Fig. 16(b). The average energy deposition increases linearly only up to about $10 \mathrm{GeV}$ and then starts becoming non-linear as also observed in the case of WA93 PMD. This non-linearity arises because of two reasons : (a) the number of shower particles does not increase linearly with energy at higher incident energies, and (b) light emission in the scintillation process is known to be non-linear when the energy deposition is high. For the present applications, however, this is not a significant problem as the cluster signal is used only for rejecting hadrons by applying a suitable threshold. This threshold, to be discussed in a later section, is rather small, not affected by non-linearity occurring at high signal levels.

The relation between the energy deposition in a pad in MeV units as given by GEANT and the ADC channel number of the CCD charge is displayed in Fig. 17. The lowest point corresponds to the energy deposition by a MIP (50 GeV $\pi^{-}$beam) and the other points correspond to electrons at different incident energies. A second order polynomial is fitted through the points to get the MeV-ADC calibration relation. This relation is used to convert the GEANT energy deposition per pad in $\mathrm{MeV}$ to ADC channels in order to generate simulated PMD data as described later. The uncertainty in the $\mathrm{MeV} \rightarrow \mathrm{ADC}$ conversion is less than $5 \%$.

\subsection{Comparison of test data and simulation}




\subsubsection{Readout resolution}

After converting the energy deposition in $\mathrm{MeV}$ obtained from simulation to ADC channels the simulation energy spectra are compared with the test beam energy spectra for various electron energies as shown in Fig. 18. The dashed histograms represent the simulation energy spectra (in ADC units) and the filled circles are the test beam data. It is observed that the width of the test beam data is larger compared to the width of the simulation energy spectra in all the cases. The increased width of the energy spectra in the test beam data is because of the extra fluctuations in the production of scintillation light, collection and transmission of light through the WLS and clear fibres and in various stages of propagation of light through the II chain to the CCD. This is in addition to the fluctuations in the energy deposition in the sensitive medium, which is well described by the GEANT simulation. For the present discussions all other contributions are termed as readout resolution. Assuming that the various contributions to the fluctuation are uncorrelated and the widths add up in quadrature, one can deduce the magnitude of contribution due to readout resolution using the relation

$$
\text { Readout Resolution }=\sqrt{\left(\frac{\sigma_{\text {test }}}{\Delta E_{\text {test }}}\right)^{2}-\left(\frac{\sigma_{\text {sim }}}{\Delta E_{\text {sim }}}\right)^{2}}
$$

where $\Delta E_{\text {test }}$ and $\Delta E_{\text {sim }}$ are the mean energy deposition obtained from test results and simulation (after applying $\mathrm{MeV}-\mathrm{ADC}$ conversion) respectively; $\sigma_{\text {test }}$ and $\sigma_{\text {sim }}$ are the widths of the distributions in the two cases.

The readout resolution as a function of cluster ADC as obtained from the test beam data is shown in Fig. 19. Only the electron data are used here as the preshower energy spectra have almost gaussian shapes. A polynomial fit is made to the above values of readout resolution for extrapolation to intermediate values of the cluster ADC. For cluster ADC values less than 3 MIPs, the shape of the energy spectrum is close to a Landau distribution. For these cases the GEANT produced MIP distribution is numerically convoluted with a gaussian function. The resulting distribution is matched with the MIP spectrum from the test beam data by adjusting the parameters of the gaussian function. The final parameters of the gaussian function are taken to represent the readout resolution.

\subsubsection{Procedure for generating simulated data}

One of the important goals of the test beam studies is to learn in detail about the detector and the readout cameras so that the GEANT results in terms of energy deposition can be converted to the pad ADC level. This data is then very similar to the actual experimental data on lead ion interactions and can be treated in identical manner to study specific physics issues. In particular the efficiency of photon counting, fraction of contaminants and associated statistical and systematic errors are all computed by a detailed comparison of simulated data and the original particle distribution obtained from an event generator.

The steps involved in generating the simulated data from the event generator (or from single particle inputs, as the case may be) are shown in the flowchart of Fig. 20. 
The package GWA98 implements detailed geometries of various detector components in the WA98 experiment within the frame work of GEANT simulation. For the PMD, the support structure with rigid frames, angular bends and the stand were all included. These are described in Ref. [11].

Using the $\mathrm{MeV}-\mathrm{ADC}$ calibration relation the energy deposited in the scintillator pad in simulation is converted to ADC channels, equivalent to experimental data. After this the ADC value is convoluted with a gaussian function of proper width taken from the readout resolution curve. If the energy deposition is less than 3 MIPs, a Landau distribution is used for convolution. The ADC value is then redistributed to neighbouring pads according to the information on the light level, probability of the number of pads fired and the fractional light leakage going into those pads as described in detail in Sec. 5.1.3. At this stage the simulated data looks very similar to the experimental data.

\subsubsection{Results of comparison}

The MIP spectrum, obtained by simulating the response of $50 \mathrm{GeV} \pi^{-}$incident on the PMD after folding all the effects, is superimposed on Fig. 15 for comparison with the test beam results. Both the shape and the mean value of $\sim 38$ ADC channels compare very well with the test data.

The simulated preshower energy spectra corresponding to the test beam, after correcting for all effects as mentioned in earlier sections, are superimposed as continuous histograms on Fig. 18 for comparison. The widths are now larger compared to the uncorrected GEANT results (dashed histograms). The agreement with the test data is very good.

The average number of pads fired and the mean cluster ADC, after applying above corrections, are compared with the test beam data in Fig. 16. In all the cases agreement between the test beam and the simulated data is excellent. These comparisons reflect the success of the methodology adopted to generate the simulated data.

\subsection{MIP in lead beam data}

As all the pads of the PMD could not be exposed to the test beam, it is desired to have an alternative method to investigate the response of MIPs in individual pads. This is important for comparing relative pad gains and normalization of camera gains. The following procedure is adopted to obtain the MIP signal in the PMD from the experimental data on $\mathrm{Pb}+\mathrm{Pb}$ collisions, where most of the produced charged hadrons appear as MIPs.

A MIP cluster in test data satisfies the criteria :

number of pads in the cluster $\leq 4$,

fraction of total signal in the hit pad $\geq 0.7$, total cluster ADC $<200$. 
Isolated clusters satisfying the above criteria in the simulated $\mathrm{Pb}+\mathrm{Pb}$ collision data have been selected as MIP signals. It is found out from the simulation study that this method works quite well to identify MIPs even in the very high particle density environment of the lead ion experiment. The mean and the shape of the distribution agree well with test data. The admixture of photons in the MIP sample of simulated lead ion data is less than $20 \%$. This suggests that the algorithm for MIP selection is satisfactory. The MIP mean is found to be $\sim 38$ channels, in close agreement with the value obtained from the test beam data.

The same prescription is also used in the experimental lead beam data to find out the MIP signal. Fig. 21 shows the MIP spectrum obtained from experimental and simulated data of $\mathrm{Pb}+\mathrm{Pb}$ collisions at $158 \cdot A \mathrm{GeV}$. Filled circles are experimental data points and the continuous histogram represents simulated data. MIP mean in this case is also around 38 channels, as expected (Fig. 15).

\subsection{Detector uniformity}

In the final configuration of the detector the response of the pad - fibre combination further changes because of the gain variations over the CCD surface and distortion in imaging leading to non-uniform division of pixels among fibres. This variation in pad-topad gain needs to be investigated.

The gains of individual pads in the assembled detector are studied in a manner similar to that in the case of WA93 PMD [5]. The MIP signal in individual pads is obtained from lead ion data by using the MIP criteria discussed above. The mean value of MIP response for each pad is obtained by fitting the MIP ADC spectrum to a Landau distribution. By analyzing a large volume of data MIP spectra for all the pads were obtained with reasonable statistics so that the mean values are stable. The MIP value for an individual pad is normalized to a global mean of the MIP values of all the pads in a box module, this normalization representing the relative pad gain.

The relative gains of all the pads studied are plotted in Fig. 22. The fluctuation of pad-to-pad relative gain is approximately 10\%. This result is consistent with the measurement of pad responses during fabrication using the $\beta$-source. Only about $2.5 \%$ of the pads are found to have either very low or very high gains, lying outside the width of the main gaussian. These arise because of several possible imperfections in the detector

at the fabrication and assembly stages. As the fraction of such pads is not significantly large, no extra care has been taken for their treatment.

\section{Parametrization for the Entire PMD}




\subsection{Extension of the test results}

The parametrizations discussed in the previous section were derived for only one box module which was tested using $e^{-}$and $\pi^{-}$beams at various energies. For the simulation of the entire PMD it is thus necessary to extend the above parametrizations in terms of suitable normalizations so that the results are applicable for all the box modules. Light leakage has been extensively studied for all the box modules using fiducial data as described in Sec. 5.1. The procedures for extending the parametrization for MeV-ADC calibration and readout resolution to the entire detector are described below.

\subsubsection{MeV-ADC relation}

In the simplest scheme, the $\mathrm{MeV}-\mathrm{ADC}$ calibration relation can be extended to other cameras by normalizing the polynomial obtained in Fig. 17 with the relative camera gains. Mean MIP responses in different cameras, obtained using data on lead-lead collisions, can be used for estimating the relative camera gains.

However the relation at higher light intensities may not follow the normalization provided at the MIP point. To check this a procedure involving detailed intercomparison of responses in different cameras has been carried out at various light intensities using the fiducial data.

The ratio $R_{j k}^{i}(l)$ of signals (mean $\mathrm{ADC}$ values) in pairs of cameras $j, k$ have been studied for different intensities $(l)$ of input light from the flasher system. This ratio denotes the relative camera gain at a particular light intensity. The light intensity in the fiducial event is determined by the signal in a camera $(i)$ different from the pair under consideration. The value of $l$ ranges from MIP level to 2500 ADC channels. For the present study $l$ has been divided into five ranges as shown in Fig. [13.

The relative gain at MIP level is given by the ratio of MIP signals in cameras $j$ and $k$. If the relative gains at different light intensities are comparable, $R_{j k}^{i}(l) \sim R_{j k}^{i}(m i p)$. Thus one should expect that the average of the relative difference $\left[\left(R_{j k}^{i}(l)-R_{j k}^{i}(m i p)\right)\right] /$ $R_{j k}^{i}$ (mip) should be almost zero. Fig. 23(a) shows the histogram of the relative difference for a large number of combinations of $i, j, k$ studied. This is found to have mean close to zero and a standard deviation of $\sim 8 \%$, which is rather small and of the same order as the uncertainty in the MeV-ADC relation. This suggests that the relative camera gains are not very much dependent on light intensity. Thus the normalization using MIP values can be considered satisfactory for the present case.

The normalization relation for the other box modules $(j)$ is :

$$
\mathrm{ADC}_{j}=\left[\mathrm{MeV} \rightarrow \mathrm{ADC}_{i}\right] \times \mathrm{MIP}_{j} / \mathrm{MIP}_{i}
$$

where $i$ refers to the box module used in the test beam. 


\subsubsection{Readout resolution}

Data on fiducial lighting, where blue light is injected into the pad through the fiducial fibre, provide the effects of fluctuations in all the steps of light transmission through the fibre and the II chain to the CCD. Thus the width of the ADC spectrum in fiducial data $\left(\sigma_{\text {fid }}\right)$ should reflect the convolution of two major components : (a) the intrinsic fluctuation in the intensity of light produced by the flasher system $\left(\sigma_{\text {source }}\right)$ and (b) fluctuations due to all the other effects of transmission of light up to the $\operatorname{CCD}\left(\sigma_{\text {trans }}\right)$, i.e., $\sigma_{\text {fid }}^{2}=\sigma_{\text {source }}^{2}+\sigma_{\text {trans }}^{2}$. The fluctuations in the production of light (i.e., in energy deposition and scintillation processes), which do not contribute to the widths of fiducial data, should be similar in all the modules as the same scintillator has been used for the fabrication of the entire detector.

As the contribution of fluctuation in input light $\left(\sigma_{\text {source }}\right)$ is essentially the same for all the cameras, the difference $\sigma_{\text {fid }}^{2}(j)-\sigma_{\text {fid }}^{2}(k)$ for any pair $j, k$ of cameras measures the difference in fluctuation due to transmission including the effect of camera to camera

variations. Fig. 23(b) shows the histogram of the deviation in width $\sqrt{\left|\sigma_{\text {fid }}^{2}(j)-\sigma_{\text {fid }}^{2}(k)\right|}$. This has a mean of only $5.6 \%$, suggesting that the parameters of readout resolution are almost independent of camera gains and can be taken to be identical for all the cameras.

\subsection{Comparison of simulated and experimental data on $\mathrm{Pb}+$ $\mathrm{Pb}$ collisions}

The usefulness of the extended parametrization for the entire PMD can be tested by comparing the results of a full simulation using the VENUS event generator and the experimental data on lead ion collisions. It is well understood that particle production in lead ion collisions need not be fully described by VENUS in all its details, but some general features of particle emission may not be very different. Thus the average pad occupancy and total energy deposition in simulated and experimental data can still be compared.

Fig. 24 shows (a) the number of pads fired and (b) the total ADC in box modules of different pad sizes for the experimental and simulated data on $\mathrm{Pb}+\mathrm{Pb}$ collisions. For a given pad size the values in the figure denote the average over the number of such box modules used. The agreement between the experimental and simulated data is within $10 \%$ for both the number of pads fired and the total ADC. This is very encouraging particularly in view of the fact that neither any parameter of the event generator was tuned nor the raw experimental data was treated in any manner except noise filtering. This suggests that the procedure for generating the simulated data and the extended parametrization are quite satisfactory. 


\section{Photon Counting}

Using the experimental data, either recorded in mode 3 (fibre or pad coordinates) or converted to mode 3 using pixel-to-fibre maps, photon counting is done by first clustering the pad hits and then applying a suitable threshold to reject hadrons. The clustering algorithm described in Ref. [5] has been used here with some modifications. Because of the use of pads of different sizes, the pad matrix over the entire detector cannot be represented by a single two-dimensional matrix to allow processing of all the pad hits in one pass. Hence the hits are clustered separately for each box module. Some tracks may produce more than one cluster either because of upstream conversion or because of splitting at the boundaries of the box modules. For photon tracks, the cluster with the higher signal is assigned a photon identity and the other one is treated as a contaminant.

Although most of the hadrons behave like MIPs, a small fraction undergoing interaction in lead converter produces signals which are similar to those of photons. Therefore all the hadrons are not rejected by applying a threshold. The exact particle identification of each hit cannot be ascertained in the experimental data. Hence the number of clusters remaining above the hadron rejection threshold is termed as $\gamma$-like. This contains majority of photons and some contaminants which reduce the purity of the photon sample.

We define the following two variables :

$$
\begin{aligned}
& \epsilon_{\gamma}=N_{c l s}^{\gamma, t h} / N_{\gamma}^{i n c} \\
& f_{p}=N_{c l s}^{\gamma, t h} / N_{\gamma-\text { like }}
\end{aligned}
$$

where $\epsilon_{\gamma}$ is the photon counting efficiency and $f_{p}$ is the fractional purity of the photon sample. $N_{\gamma}^{\text {inc }}$ is the number of incident VENUS photons on the PMD, $N_{c l s}^{\gamma, t h}$ is the number of photon clusters above the threshold and $N_{\gamma-l i k e}$ is the total number of clusters above the threshold.

An estimate of the optimum value of hadron rejection threshold and the achievable purity of the photon sample along with the estimates of photon counting efficiency is made by a detailed study of the simulated data, which are generated using VENUS event generator and GEANT simulation following the scheme of the flowchart of Fig. 20. No lower threshold on the energy spectrum of photons is applied.

By adjusting the discrimination threshold it is possible to obtain a reasonably pure sample of photons, although some contaminants always remain. Fig. 25 shows the photon counting efficiency and the purity as a function of hadron rejection threshold (in MIP units) for two different centralities of $\mathrm{Pb}+\mathrm{Pb}$ collisions. The centrality of the reaction is defined by the transverse energy $\left(E_{T}\right)$ obtained from the mid-rapidity calorimeter in the WA98 experiment [6]. Central events span the region $E_{T} \geq 330 \mathrm{GeV}$ corresponding to the top $5 \%$ of minimum bias cross section and peripheral events correspond to the region $40 \mathrm{GeV} \leq E_{T} \leq 100 \mathrm{GeV}$. In both cases the photon counting efficiency decreases with increasing threshold. The purity improves significantly with increasing threshold only up to $\sim 3$ MIPs and then rather slowly at higher thresholds. 
The estimated photon counting efficiency is dependent on both the detector hardware and the clustering software. A photon is labeled as "converted" on depositing a minimum energy equivalent to 0.2 MIP. The mean conversion probability for VENUS photons within the PMD acceptance in the case of $\mathrm{Pb}+\mathrm{Pb}$ collisions is found to be 95\%. This gives an upper limit to the photon counting efficiency when no hadron rejection threshold is applied, i.e., when all the "converted" photons can be counted. Photon counting efficiency close to this value ( $93 \%$ ) is achieved only for peripheral events with no threshold as shown in Fig. 25. The maximum value for central events is about $84 \%$. The decrease in photon counting efficiency for central events arises because of loss of clusters due to overlap in the higher multiplicity environment of central collision events.

For all practical purposes a 3 MIP threshold appears as an optimum choice for hadron rejection leading to reasonable values for both the photon counting efficiency and the purity. With this, the photon counting efficiencies for central and peripheral cases are $68 \%$ and $73 \%$, respectively. The purity of the photon sample in the two cases are $65 \%$ and $54 \%$, respectively.

The efficiency values are comparable to or better than that of the WA93 PMD. The PMD is therefore quite suitable to handle the increased particle density. The purity of the photon sample is somewhat lower in the present case. The purity depends on the relative population of photons and hadrons as given by the event generator. The ratio $N_{\gamma} / N_{\text {charge }}$, on the PMD acceptance for central collisions is found to be only $\sim 0.7$ in the present case compared to $\sim 0.8$ for the WA93 case. This difference partly explains the lower purity in the present case. A detailed investigation of the centrality and pseudorapidity dependence of photon counting efficiency and purity will be presented in a subsequent publication.

For the experimental data one is able to determine only $N_{\gamma-l i k e}$, the number of clusters above hadron rejection threshold. Using the estimated values of $\epsilon_{\gamma}$ and $f_{p}$, one can estimate the number $\left(N_{\gamma}^{e s t}\right)$ of photons incident on the detector in the event using the relation :

$$
N_{\gamma}^{e s t}=N_{\gamma-l i k e} \cdot f_{p} / \epsilon_{\gamma}
$$

Statistical error on $N_{\gamma}^{e s t}$ thus deduced is governed mainly by the nature of counting statistics. In the present case the values obtained for the statistical error are $4.6 \%$ for central collision events, where the average number of incident VENUS photons, $\left\langle N_{\gamma}^{\text {inc }}\right\rangle$, is 428 , and $11 \%$ for peripheral collision events having $<N_{\gamma}^{i n c}>\sim 116$. Details regarding the systematic errors will be discussed in a future communication.

Recently it has been reported [12] that using neural network techniques and considering the full information of preshower profile, much better discrimination between photons and hadrons can be achieved. Thus one can improve the purity of the photon sample without sacrificing the photon counting efficiency. In this method each cluster is assigned an output value optimized by the network algorithm and one can select photons and hadrons by applying suitable threshold on the network output spectrum. Full potential of this method and its application to actual experimental data is still under investigation and will be reported in subsequent publications. 


\section{Summary}

The preshower PMD for the WA98 experiment has been fabricated and tested. The detector consists of $3 \mathrm{X}_{0}$ thick lead converter plates and a matrix of plastic scintillator pads placed behind the converter in 28 box modules. Square pads of four sizes varying from $15 \mathrm{~mm}$ to $25 \mathrm{~mm}$ have been employed. Scintillation light is transmitted to the readout device using a short WLS fibre spliced with a long EMA-coated clear fibre to improve light transmission. Each box module consists of a matrix of $38 \times 50$ pads and is read out using one II + CCD camera system obtained from the UA2 experiment. The detector extends to $21 \mathrm{~m}^{2}$ in area and covers the pseudorapidity region $2.5 \leq \eta \leq 4.2$ of which the region $3.2 \leq \eta \leq 4.0$ has full azimuthal coverage.

The imaging of the fibres on the CCD surface has been greatly improved by several modifications in the techniques of detector fabrication and assembly. This has resulted in fewer number of pads fired for a preshower of a given electron energy as compared to that in the case of WA93 PMD. In addition, the granularity has been optimized so that the pad occupancy and the multiple hit probability are quite low. These efforts have improved the performance of the detector in the much higher particle density environment of lead ion collisions.

The detector was tested using electron and pion beams at different energies to study the preshower behaviour and the response of minimum ionizing particles. Data were also taken using fiducial lighting at several light intensities for all the readout units in the box modules. The test beam and fiducial data have been used to deduce the parameters describing the behaviour of the detector. A simulation framework incorporating these parameters has been developed to generate simulated data which are very similar to the experimental data and can be compared directly to study the physics with event generators. The simulated and the experimental data compare very well for both the test beam runs and for gross features of the lead beam runs.

The photon counting efficiency is found to be $68 \%$ for the central and $73 \%$ for the peripheral $\mathrm{Pb}+\mathrm{Pb}$ collision events as given by the VENUS event generator. The slight decrease in the efficiency for central collision case is due to loss of clusters because of overlap. The purity of the photon sample is about $65 \%$ for central and $54 \%$ for peripheral cases.

\section{Acknowledgements}

We gratefully acknowledge the financial support of the Department of Atomic Energy, the Department of Science and Technology and the University Grants Commission of the Government of India. Two of us (DSM and NKR) are grateful to the Council of Scientific and Industrial Research for the financial support for carrying out this work. We express our sincere gratitude to CERN authorities for the loan of UA2 readout cameras and for extending the servicing facilities for them. We thank C. Engster and J. Dupont for help in testing the UA2 cameras and P. Pierre and T. Reynes for the engineering support during the installation of the PMD. We are grateful to the PPE Division of CERN for providing local hospitality to the Indian team and to the Indo-FRG Exchange Pro- 
gramme for supporting the visits of collaborators from GSI, Germany to India. We also acknowledge the cooperation of the engineering, computer and other support staff at the collaborating institutions (VECC, IOP, Jammu University, Panjab University and Rajasthan University) during the period of design, fabrication and assembly of this detector. We express our sincere gratitude to the CERN SPS crew for providing good beams during test runs.

\section{References}

[1] E.L. Feinberg, Nuovo Cimento A34 (1976) 391;

E.V. Shuryak, Phys. Lett. B79 (1978) 135;

D.K. Srivastava and B. Sinha, Phys. Rev. Lett. 73 (1994) 2421.

[2] A.A. Anselm and M.G. Ruskin, Phys. Lett. B266 (1991) 482;

J.D. Bjorken, K.L. Kowalski, C.C. Taylor, "Baked Alaska", SLAC-PUB-6109 (1993).

J.-P. Blaizot and A. Krzywcki, Phys. Rev. D46 (1992) 246.

K. Rajagopal and F. Wilczek, Nucl. Phys. B399 (1993) 395.

[3] C.M.G. Lates, Y. Fujimoto, and S. Hasegawa, Phys. Rep. 65 (1980) 151.

Y. Takahasi et al., (JACEE Collab.) Proc. $7^{t} h$ Int'l. Symp. on Very High Energy Cosmic Ray Interactions, (1992), Ann Arbor, Michigan, ed. L. Jones.

[4] H. Baumeister et al, Nucl. Instr. Meth. A292 (1990) 81.

[5] M. M. Aggarwal et al., Nucl. Instr. and Meth. A372 (1996) 143.

[6] Proposal for a Large Acceptance Hadron and Photon Spectrometer, H.H. Gutbrod et. al., CERN-SPSLC-91-17, CERN-P260 (1991) 87 p.

[7] R. E. Ansorge et al., Nucl. Instr. and Meth. A265 (1988) 33.

[8] K. Werner, Phys. Rep. C232 (1993) 87.

[9] R. Brun et al., GEANT3 user's guide, CERN/DD/EE/84-1 (1984).

[10] M. R. Dutta Majumdar et al., DAE Symp. Nucl. Phys. (Calicut, India), 36B (1993) 410; DAE Symp. Nucl. Phys. (Bhubaneswar, India), 37B (1994) 483; Int'l. Nucl. Phys. Symp, (Bombay, 1995) Book of Abstracts, I-45.

[11] B.K. Nandi, Thesis, under preparation.

[12] S. Chattopadhyay et al, preprint hep-ex/9807012, submitted to Nucl. Instr. Meth. 


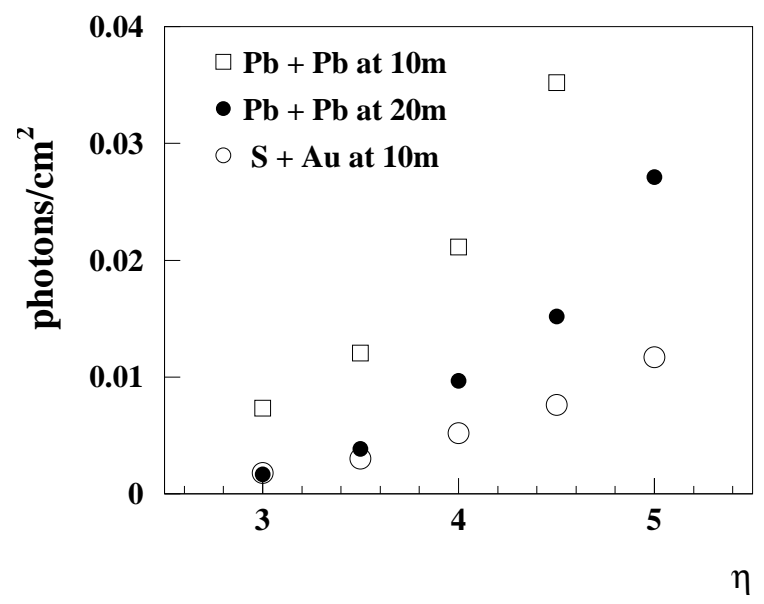

Figure 1: Particle density (photon $/ \mathrm{cm}^{2}$ ) as a function of pseuedorapidity $(\eta)$ in central lead-lead collisions as given by VENUS.

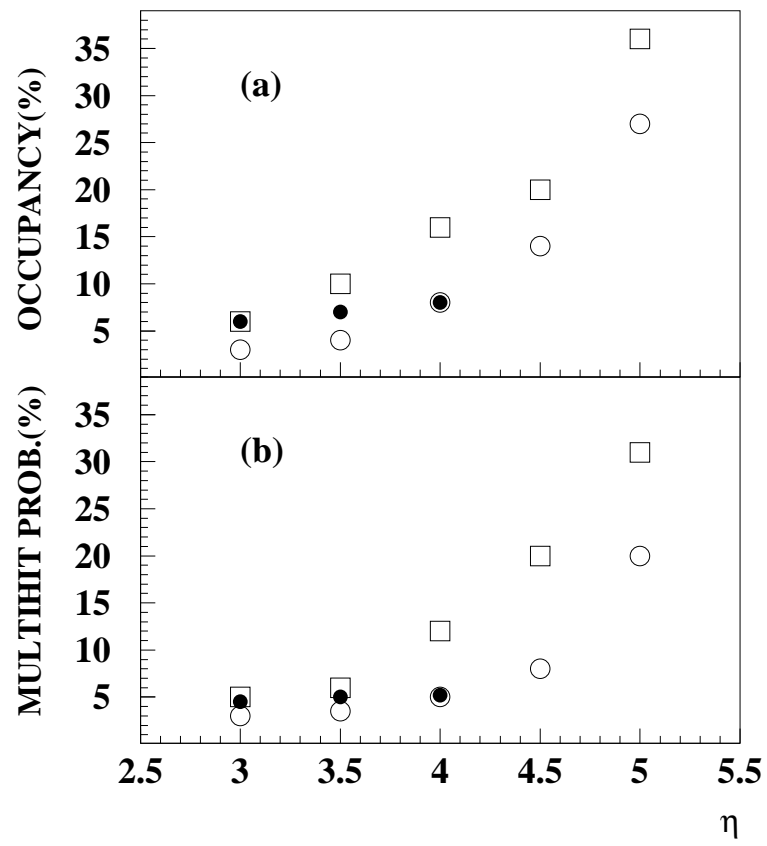

Figure 2: (a) Occupancy and (b) multiple hit probability for three different cases: 15 mm pads (open circles), $25 \mathrm{~mm}$ pads (open squares) and the final configuration having four different pad sizes (filled circles). The distance from the target was $21.5 \mathrm{~m}$ for the final configuration and $20 \mathrm{~m}$ for the other cases. 


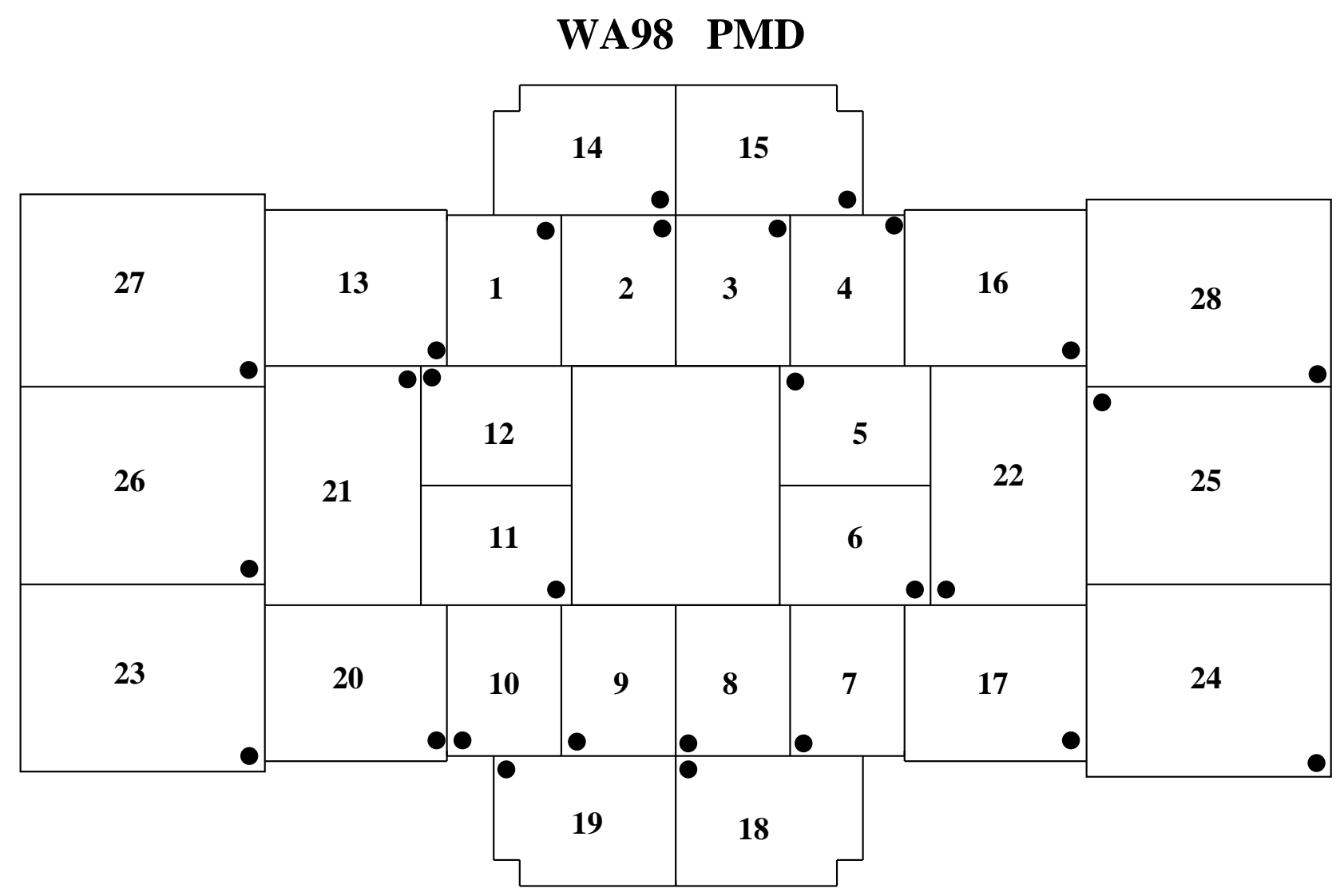

Box numbering scheme as viewed from the ZDC

Figure 3: Layout of the detector box modules. The black dot in each box indicates the corner where the pad with the co-ordinate $(01,01)$ is located. Box modules numbering 1-12 have $15 \mathrm{~mm}$ pads, $13-20$ have $20 \mathrm{~mm}$ pads, $21-22$ have $23 \mathrm{~mm}$ pads and the rest have $25 \mathrm{~mm}$ pads.

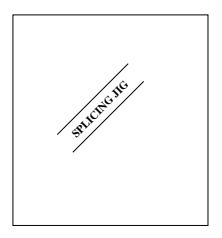

Figure 4: Schematic of the splicing jig arrangement. 


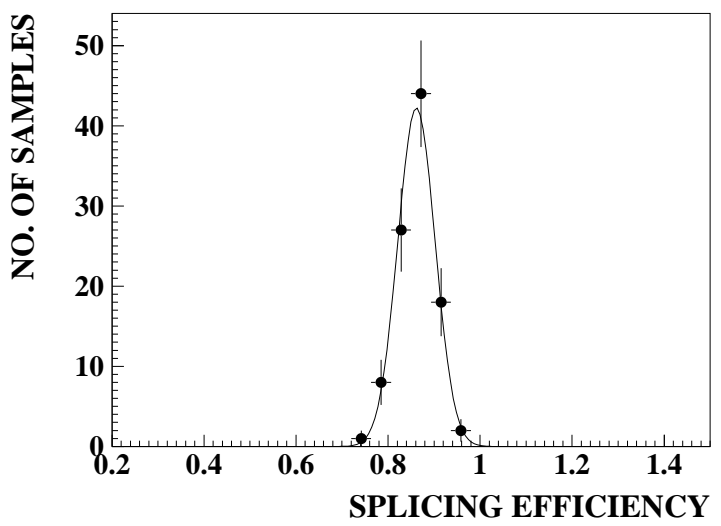

Figure 5: Splicing efficiency ( fraction of light transmitted across the joint) for the case of $8 \mathrm{~cm}$ long WLS fibre joined with $192 \mathrm{~cm}$ long clear coated fibre.

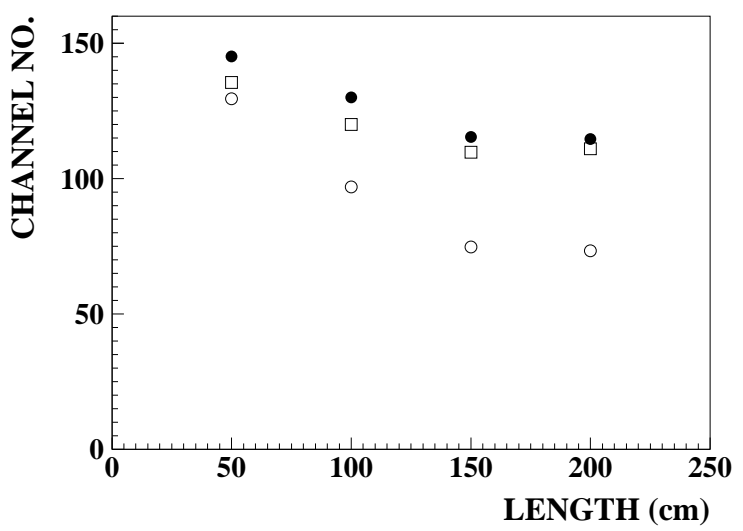

Figure 6: Light output of the scintillator pads (expressed as peak channel number of the digitized PMT signal) as a function of fibre length in three different cases : open circles represent the pads with full length WLS fibre, open squares represent the pads having 8 $\mathrm{cm}$ long WLS fibre piece spliced with clear coated fibre, and filled circles represent the pads with WLS $(8 \mathrm{~cm})+$ clear fibre and reflector paint applied to the tip of the WLS fibre inside the pad. 


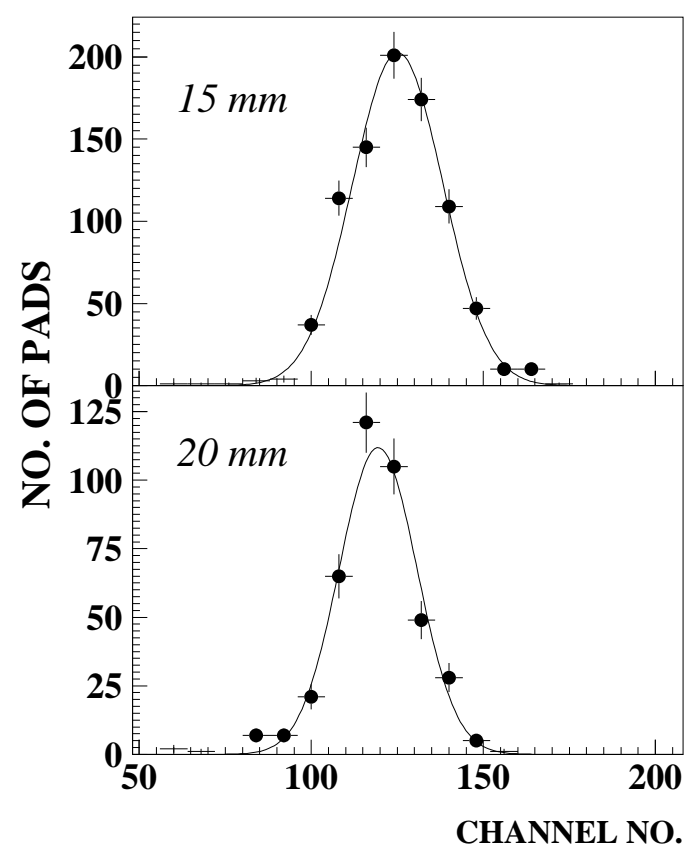

Figure 7: Response of pads to ${ }^{106} \mathrm{Ru} \beta$-rays. Filled circles are the data points showing distributions of peak channel numbers of the PMT signals. Continuous lines are the gaussian fits. Pad sizes are as indicated within the boxes. 


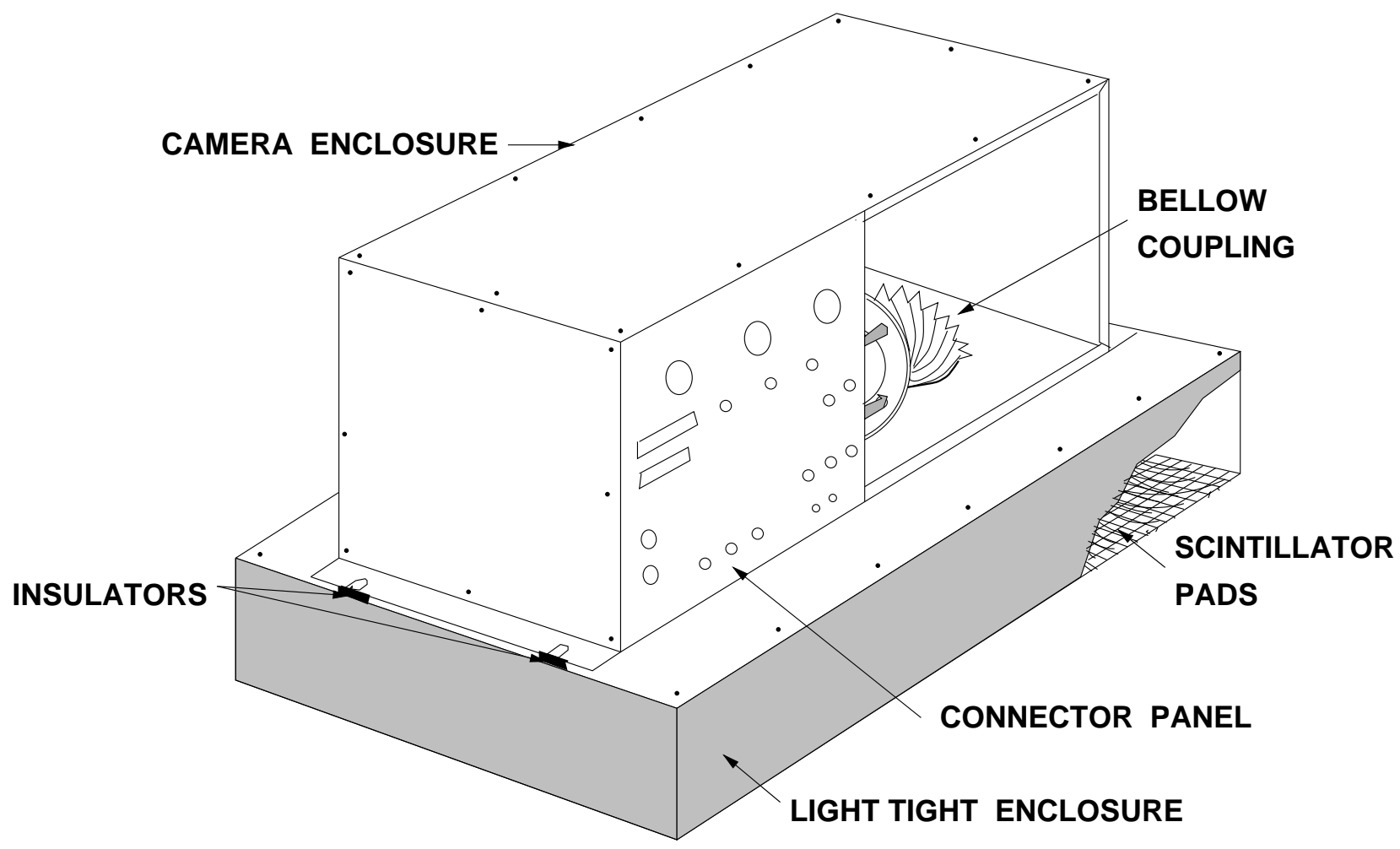

Figure 8: Sketch showing a detector box module consisting of a light tight enclosure for scintillator pads and the camera enclosure on top.
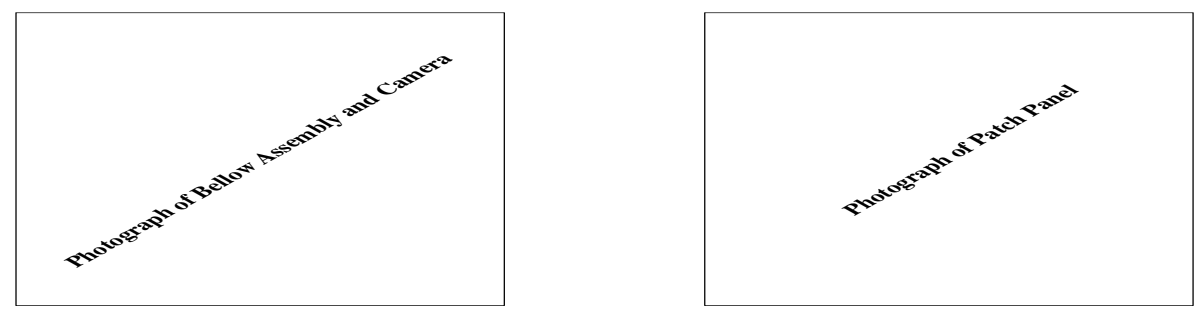

Figure 9: Photographs showing (a) a section of the detector box module with the rubber bellow and the II + CCD camera system coupled with the FEC plate, and (b) the connector panel for the electrical and optical connections. 


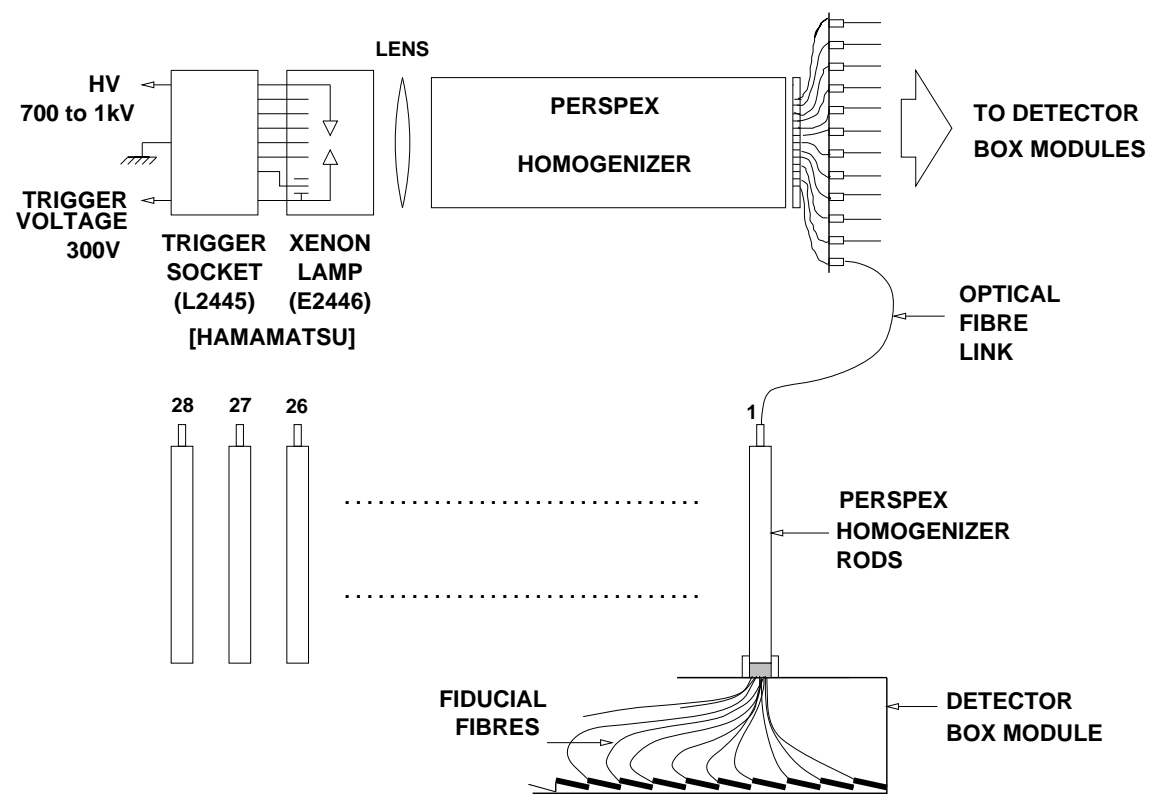

Figure 10: Schematics of Xenon flasher and the optical distribution system for injecting externally triggered light into the fiducial pads.
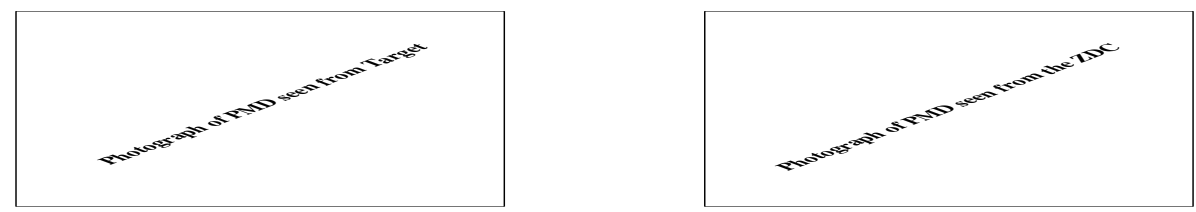

Figure 11: Photographs showing (a) the front side of the PMD, as viewed from the target, with the support structure and lead converter plates, and (b) back side of the PMD showing the detector box modules (orange colour) and the camera enclosures (blue colour).

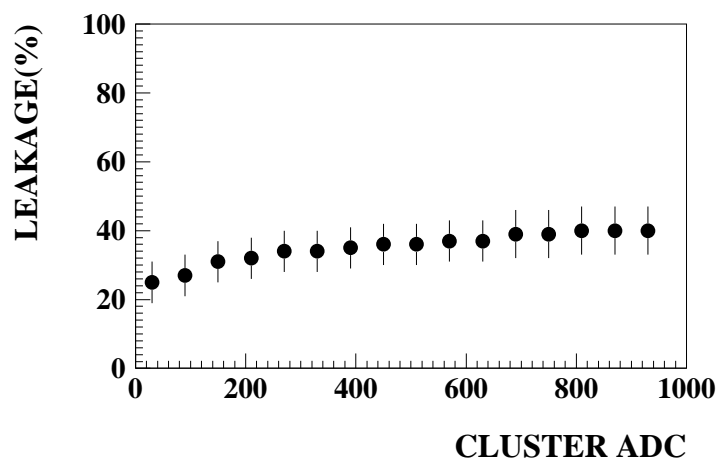

Figure 12: Percentage of light leaking outside the assigned pixels of a given fibre as a function of total light (cluster ADC). The error bars denote the r.m.s. of the distribution of leakage. 


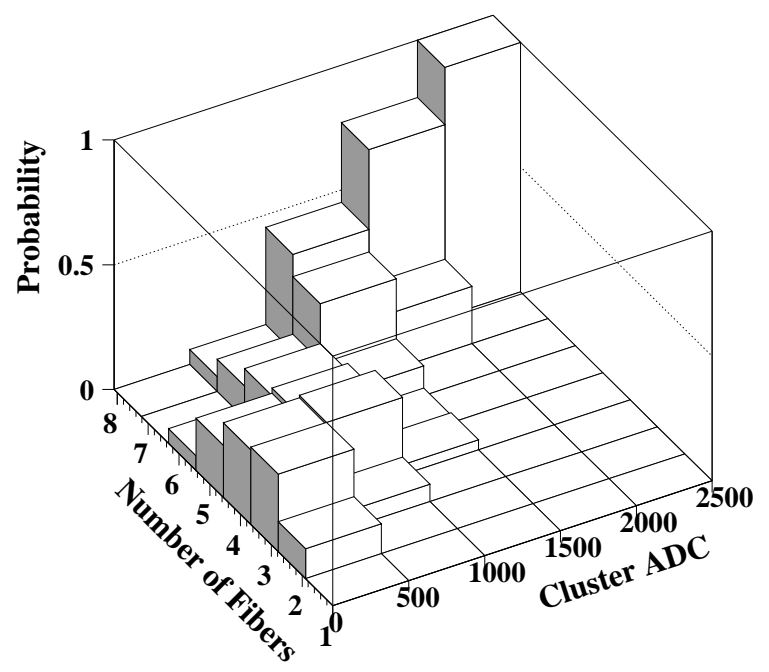

Figure 13: Probability distribution of the number of fibres surrounding the fiducial one affected by light leakage for various light intensities as denoted by the cluster ADC.

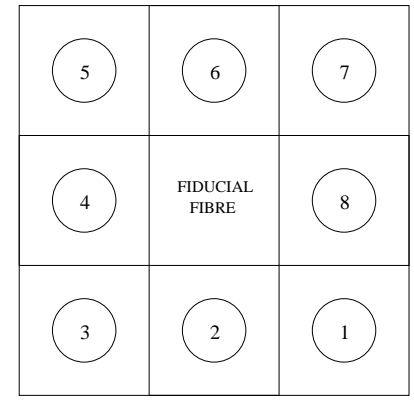

Figure 14: Position of the eight fibres surrounding the fiducial one in a rectangular matrix. This nomenclature is used in Table II, see text. 


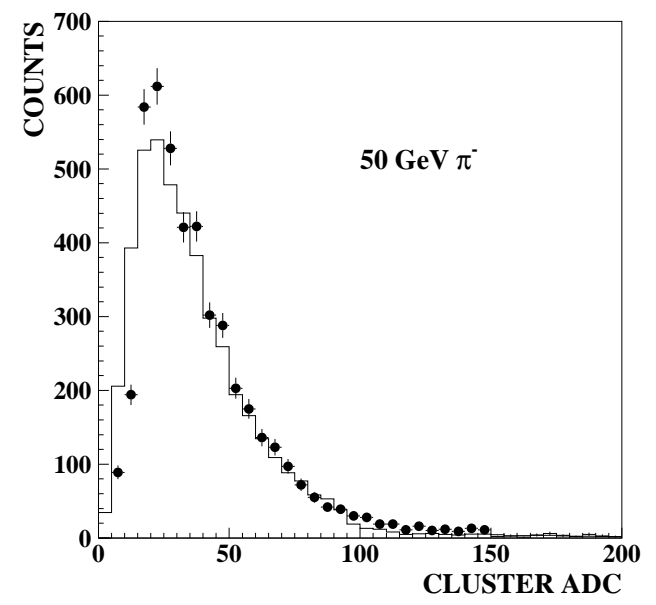

Figure 15: ADC spectrum of $50 \mathrm{GeV} \pi^{-}$passing through a PMD box module without converter. Filled circles denote the test beam data. The continuous histogram represents simulation results after using $\mathrm{MeV}-\mathrm{ADC}$ conversion and accounting for readout resolution and leakage as discussed in the text. 


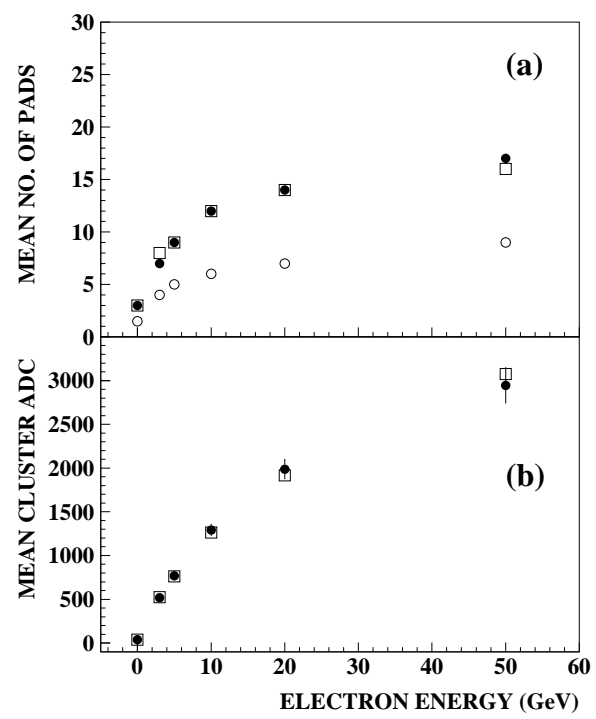

Figure 16: Preshower characteristics : (a) Mean number of pads fired as a function of incident electron energy. Filled circles denote the test beam data, open circles correspond to the GEANT simulation before light leakage to the surrounding fibres, and open squares represent the simulation results after introducing light leakage to the surrounding fibres. (b) Mean cluster ADC as a function of incident electron energy. Filled circles denote the test beam data and open squares represent the simulation results after folding all the effects. The results shown are for $15 \mathrm{~mm}$ pad sizes. The point at zero energy represents the MIP case. 


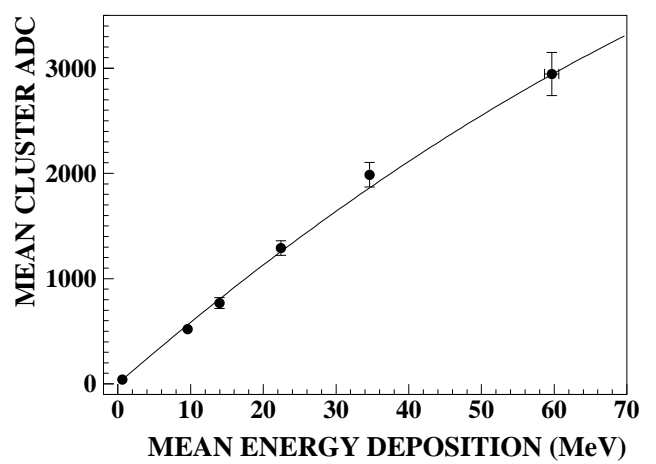

Figure 17: Mean cluster ADC of the test beam data vs. the mean energy deposition from GEANT simulation. The lowest point corresponds to $50 \mathrm{GeV} \pi^{-}$(a MIP) and other points correspond to electrons at incident energies of 3, 5, 10, 20 and $50 \mathrm{GeV}$. The continuous curve is a polynomial fit to the data and represents the MeV-ADC calibration relation. 


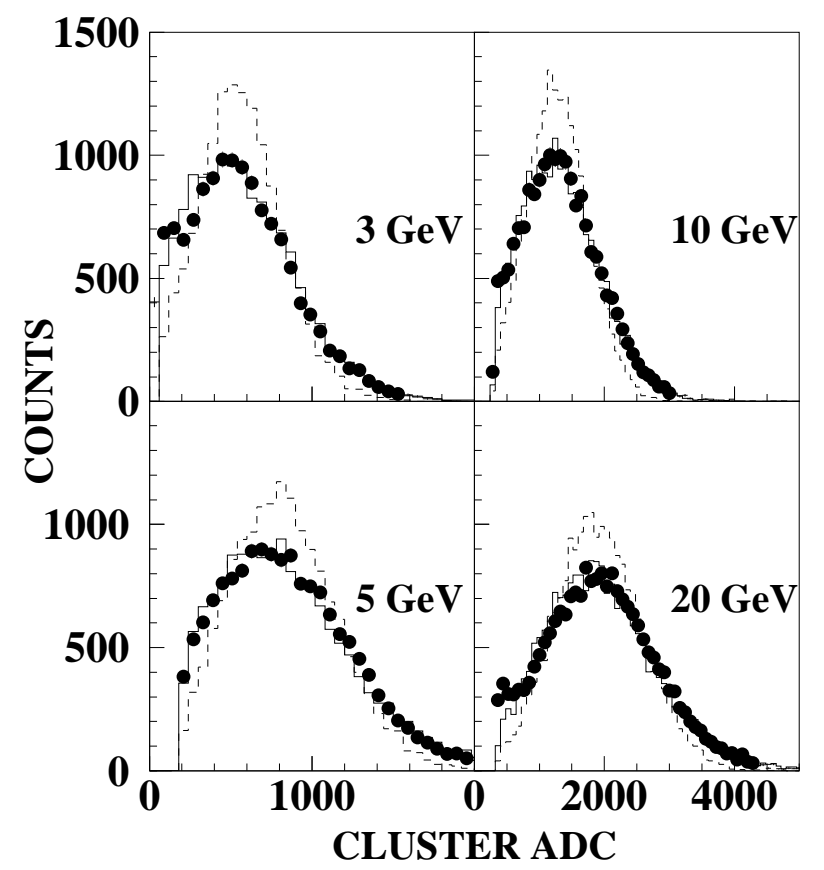

Figure 18: The preshower energy spectra for electrons at different energies as indicated. The filled circles denote the test beam data. The dashed histograms represent the simulated spectra before readout resolution and the continuous histograms represent the simulated spectra after folding the effects of readout resolution.

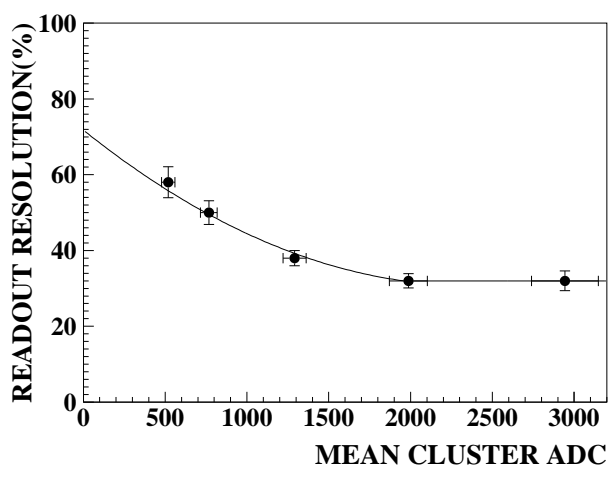

Figure 19: Readout resolution as a function of mean cluster ADC. The continuous line represents a second order polynomial fit to the data points up to $2000 \mathrm{ADC}$ and a straight line fit beyond 2000 ADC. 


\section{PMD Simulation}

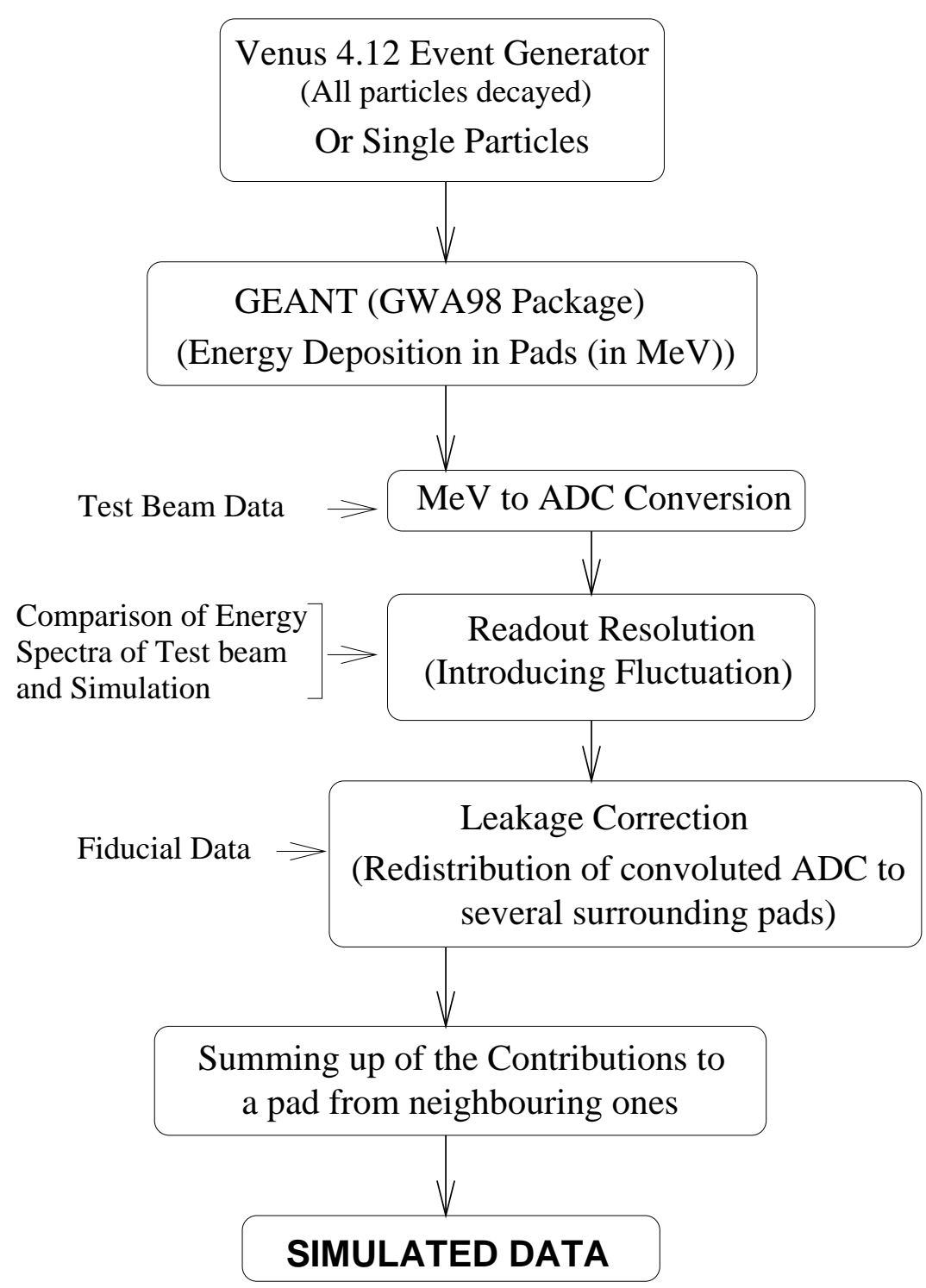

Figure 20: Flow chart showing various steps in generating the simulated data. 


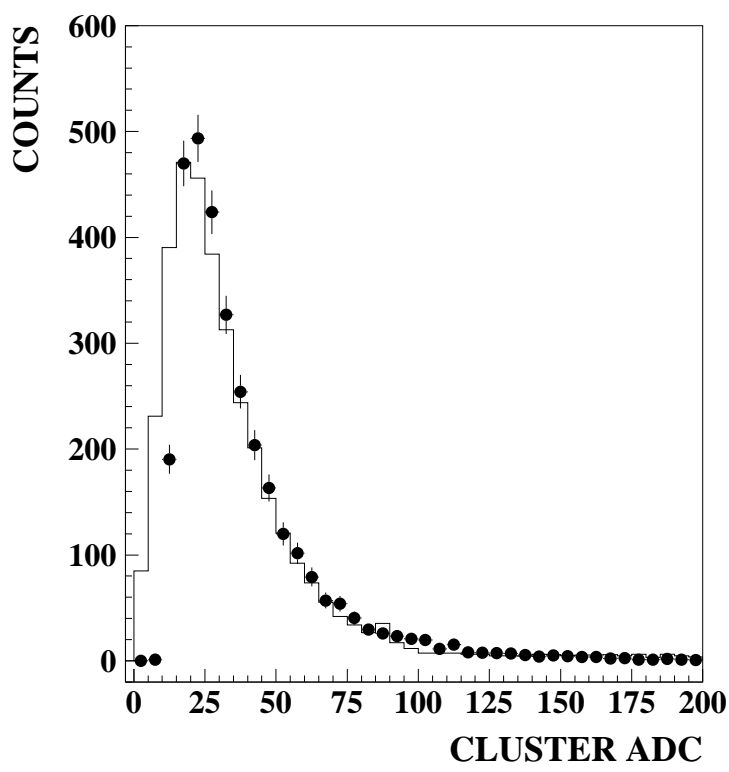

Figure 21: The MIP spectra derived from the experimental data on lead-lead collisions (filled circles) and from the simulated data (continuous histogram).

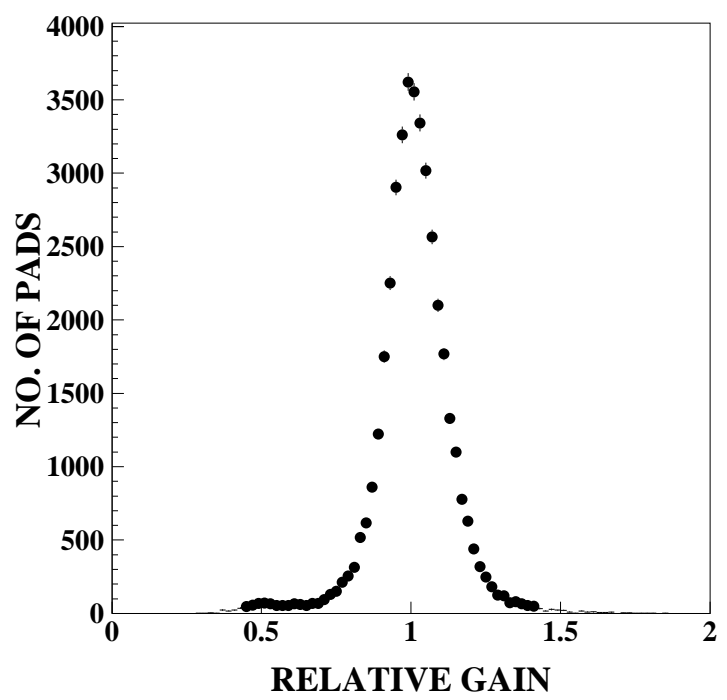

Figure 22: Distribution of relative pad gains for the entire detector. The width $(\sigma)$ of the main gaussian is $10 \%$. 

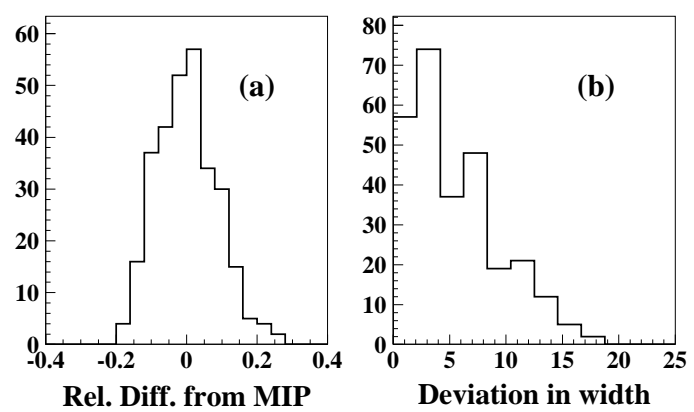

Figure 23: Histograms of (a) the relative difference of the ratio of signals in pairs of cameras at different light intensities to that at MIP light level as defined in the text and (b) the deviation in widths for pairs of cameras at different light intensities.

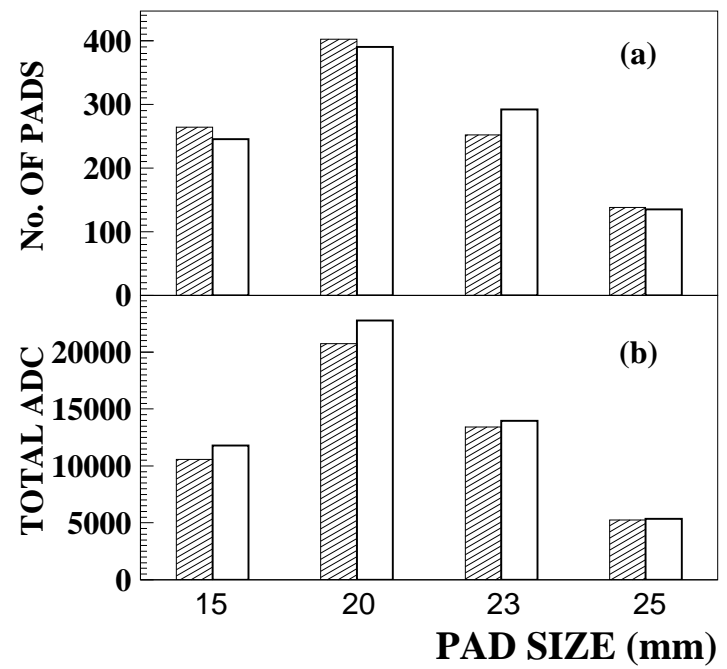

Figure 24: Comparison of experimental and simulated data on $\mathrm{Pb}+\mathrm{Pb}$ collisions for box modules of different pad sizes : (a) number of pads fired and (b) total ADC in an event. The shaded bars denote the experimental data. 


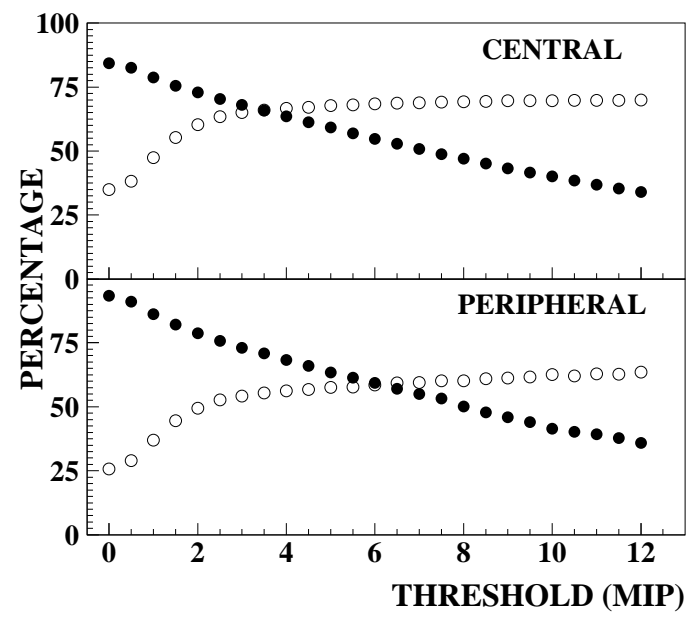

Figure 25: Variation of the photon counting efficiency (filled circles) and the fractional purity (open circles) with hadron rejection threshold (in MIP units). Top part shows the case of central events and the bottom part is for the peripheral events. 